\title{
Epigenetic dysregulation of $O x t r$ in Tet1-deficient mice has implications for neuropsychiatric disorders
}

\author{
Aaron J. Towers, ${ }^{1}$ Martine W. Tremblay, ${ }^{1}$ Leeyup Chung, ${ }^{2}$ Xin-lei Li, ${ }^{2,3}$ Alexandra L. Bey, ${ }^{4}$ \\ Wenhao Zhang, ${ }^{5}$ Xinyu Cao, ${ }^{2}$ Xiaoming Wang, ${ }^{2}$ Ping Wang, ${ }^{2}$ Lara J. Duffney, ${ }^{2,4}$ Stephen K. Siecinski, \\ Sonia Xu, ${ }^{2}$ Yuna Kim, ${ }^{2}$ Xiangyin Kong, ${ }^{3}$ Simon Gregory, ${ }^{1,6}$ Wei Xie, ${ }^{5}$ and Yong-hui Jiang, ${ }^{1,4,7,8}$ \\ 'University Program in Genetics and Genomics and 'Department of Pediatrics, Duke University, Durham, North Carolina, \\ USA. ${ }^{3}$ Laboratory of Molecular Genetics, Shanghai Jiao Tong University School of Medicine, Shanghai, China. ${ }^{4}$ Department \\ of Neurobiology, Duke University, Durham, North Carolina, USA. ${ }^{5}$ School of Life Sciences, Tsinghua University, Beijing, \\ China. ${ }^{6}$ Department of Neurology and Duke Molecular Physiology Institute, ${ }^{7}$ Duke Institute for Brain Sciences, and \\ ${ }^{8}$ Program in Cellular and Molecular Biology, Duke University, Durham, North Carolina, USA.
}

OXTR modulates a variety of behaviors in mammals, including social memory and recognition. Genetic and epigenetic dysregulation of OXTR has been suggested to be implicated in neuropsychiatric disorders, including autism spectrum disorder (ASD). While the involvement of DNA methylation is suggested, the mechanism underlying epigenetic regulation of OXTR is largely unknown. This has hampered the experimental design and interpretation of the results of epigenetic studies of OXTR in neuropsychiatric disorders. From the generation and characterization of a new line of Tet1 mutant mice - by deleting the largest coding exon 4 (Tet1 ${ }^{1 e 4}$ ) - we discovered for the first time to our knowledge that Oxtr has an array of mRNA isoforms and a complex transcriptional regulation. Select isoforms of $O x t r$ are significantly reduced in the brain of Tet ${ }^{\text {te4-I- }}$ mice. Accordingly, CpG islands of Oxtr are hypermethylated during early development and persist into adulthood. Consistent with the reduced express of OXTR, Tet 1 $^{1 e 4-/-}$ mice display impaired maternal care, social behavior, and synaptic responses to oxytocin stimulation. Our findings elucidate a mechanism mediated by TET1 protein in regulating Oxtr expression by preventing DNA hypermethylation of Oxtr. The discovery of epigenetic dysregulation of Oxtr in TET1-deficient mouse brain supports the necessity of a reassessment of existing findings and a value of future studies of OXTR in neuropsychiatric disorders.

Authorship note: AJT and MWT contributed equally to this work.

Conflict of interest: The authors have declared that no conflict of interest exists.

License: Copyright 2018, American Society for Clinical Investigation.

Submitted: February 21, 2018 Accepted: October 31, 2018 Published: December 6, 2018

Reference information: JCI Insight. 2018;3(23):e120592. https://doi.org/10.1172/jci. insight.120592.

\section{Introduction}

Oxytocin, the brain's most abundant neuropeptide, acts as a neuromodulator and hormone to its G protein-coupled oxytocin receptor (OXTR) and modulates a variety of behaviors including social memory and recognition, sexual and aggressive behaviors, and bonding and maternal care (1-4). OXTR is a small gene, with only 4 exons, and the coding exon spans only a portion of exons 3 and $4(5,6)$. The expression of $O X T R$ in mammals is cell type specific and developmentally regulated $(7,8)$. The expression of $O X T R$ is higher during parturition and lactation in the uterus and mammary tissues, respectively, as well as in the brain during mating, social bonding, and child rearing (2). The mechanism underlying this dynamic transcriptional regulation is poorly understood. The involvement of DNA methylation in regulating the expression of OXTR has been suggested, but the exact mechanism mediating this process remains largely unknown (9-11). Epigenetic studies in humans have found altered DNA methylation in the 5' CpG island of $O X T R$ in a wide spectrum of morbid behaviors and neuropsychiatric disorders, such as autism spectrum disorder (ASD), schizophrenia, bipolar, depression, obsessive-compulsive disorders (OCD), and anxiety (12-18). Similar correlations between DNA methylation and behavior are also reported in other species, such as dog and nonhuman primates $(19,20)$.

The discovery of the ten-eleven translocation (TET) family of methylcytosine dioxygenases has shed light on the regulation of DNA methylation during the development $(21,22)$. TET proteins catalyze active demethylation of DNA in a stepwise process through formation of 5-hydroxymethylcytosine (5hmC) and 
other oxidative derivatives of 5 -methylcytosine $(5 \mathrm{mC})(23,24)$. The mouse Tet gene family $($ Tet $1-3)$ is differentially regulated during development and in adult brain (25). Tet1 expression is relatively enriched in embryonic stem cells (ESCs) $(25,26)$ and has been implicated in regulating genes important for cellular differentiation, pluripotency, and neurogenesis (27-29). In addition to being a DNA demethylation intermediate, $5 \mathrm{hmC}$ is a stable DNA modification and is present at variable levels in ESCs and adult tissues, but it is specifically enriched in postnatal brains (30-32), where it accumulates with age (33). The primary role of $5 \mathrm{hmC}$ and TET protein is believed to contribute to transcriptional regulation of the genes that are important for the brain development and function (34-41). However, the exact molecular mechanism underlying these processes has not been completely elucidated. One hypothesis for brain enrichment of $5 \mathrm{hmC}$ is that key neural genes important for brain function are regulated by TET-mediated hydroxymethylation. Identification of such genes would be the first critical step to investigate the function of TET proteins and $5 \mathrm{hmC}$ in the brain.

Three lines of Tet 1 mutant mice that disrupt different portions of coding exons have been reported (42-44). Studies of these mutant mice have identified several neural genes that are dysregulated in Tet1-deficient mouse brains $(42,43)$. However, the findings from these studies of Tet 1 mutant mice reveal some similarities, as well as significant differences. The reasons responsible for these differences are not immediately clear. A recent report of different Tet 1 isoforms due to the alternative splicing during the early development may suggest that mutations targeting different exons could have different molecular consequence $(37,42,45,46)$.

From the generation and analysis of a new line of Tet1 mutant mice, we discovered that the expression of the Oxtr gene was significantly reduced in the brain of TET1-deficient mice. Unexpectedly, we uncovered that Oxtr displays a complex transcriptional regulation and an array of mRNA isoforms in the brain. The reduced expression of Oxtr only affected select mRNA isoforms in Tet1-deficient brains. Accordingly, the CG sites within the 5' CpG island of Oxtr was selectively hypermethylated in embryonic and postnatal brains. Thus, we provide the first evidence to our knowledge of an epigenetic mechanism capable of directly regulating Oxtr expression in vivo. These discoveries provide a molecular framework for reinterpreting findings from existing genetic and epigenetic studies and, more importantly, are valuable to guide the experimental design of future studies of the transcriptional regulation and the role of $O X T R$ in neuropsychiatric disorders.

\section{Results}

Generation of a line of Tet1 mutant mice and homozygous ESCs. Previous studies have reported 3 lines of Tet1 mutant mice that delete coding exon 5, exons 11-13, or by inserting a gene trap (Gt) in intron 2 (44) (Figure 1A). We generated a new line of Tet 1 mutant mice by targeting and floxing the largest coding exon, exon 4 (Figure 1A and Supplemental Figure 1A; supplemental material available online with this article; https://doi.org/10.1172/jci.insight.120592DS1). Deletion of exon 4 causes a frameshift, leading to a premature stop codon in exon 6 that is 36 amino acids after the end of exon 3 and results in disruption of the catalytic domain of TET1. Expression analysis by reverse transcription PCR (RT-PCR) confirmed exon 4 deletion (Figure 1B, right panel), and Western blot analysis showed deficiency of TET1 protein from Tet $1^{\text {1et-I- }}$ mouse hippocampus (Figure 1C). TET1 has been suggested to promote transcription of pluripotency factors by an in vitro study using RNAi and plays a distinct role during the different stages of the development $(47,48)$. Therefore, we generated homozygous Tet $1^{4 e 4 /-}$ ESCs by targeting the second allele of Tet $1^{e 4 f}$ ESCs sequentially, followed by introduction of Cre in Tet $1^{e f f / f}$ ESCs (Figure 1D and Supplemental Figure 1, B-E). Tet $1^{\text {set-1- }}$ ESCs are viable with no apparent abnormality in cellular morphology. Tet1 and Tet2, but not Tet3, are known to be highly expressed in ESCs $(25,26)$. Accordingly, we found Tet2 - but not Tet3 - upregulated in Tet $1^{\text {te4-/ }}$ ESCs (Figure 1E), suggesting a compensatory mechanism of Tet2 in response to deficiency of Tet1 in ESCs. However, this similar compensatory mechanism was not observed in adult hippocampus (Figure 1F).

Tet $1^{\text {1e4+/- }}$ mice were backcrossed to C57BL/6J mice for more than 6 generations (N6) before molecular experiments and behavioral analysis. Tet $1^{1 e 4+/-}$ mice on a C57BL/6J background displayed partial perinatal lethality and a deviation from th expected Mendelian ratio of genotypes at weaning age, indicating a role for TET1 in early development (Table 1). The surviving Tet $1^{1 e 4-/-}$ mice showed a mild degree of growth retardation, as Tet $1^{\text {tet-1- }}$ mice weighed significantly less than $T e t 1^{+/+}$littermates at weaning; this persisted into adulthood (Figure $1 G$ ). Tet $1^{\text {tet-I- }}$ mice did not have any apparent abnormal behavior in their home cage.

Transcriptional dysregulation of neural genes in Tet $1^{\text {ted-1- }}$ mutant mice. While $5 \mathrm{hmC}$ is abundant in the postnatal brain, the exact regulatory function of this epigenetic mark remains less understood $(30,31,49)$. 
A

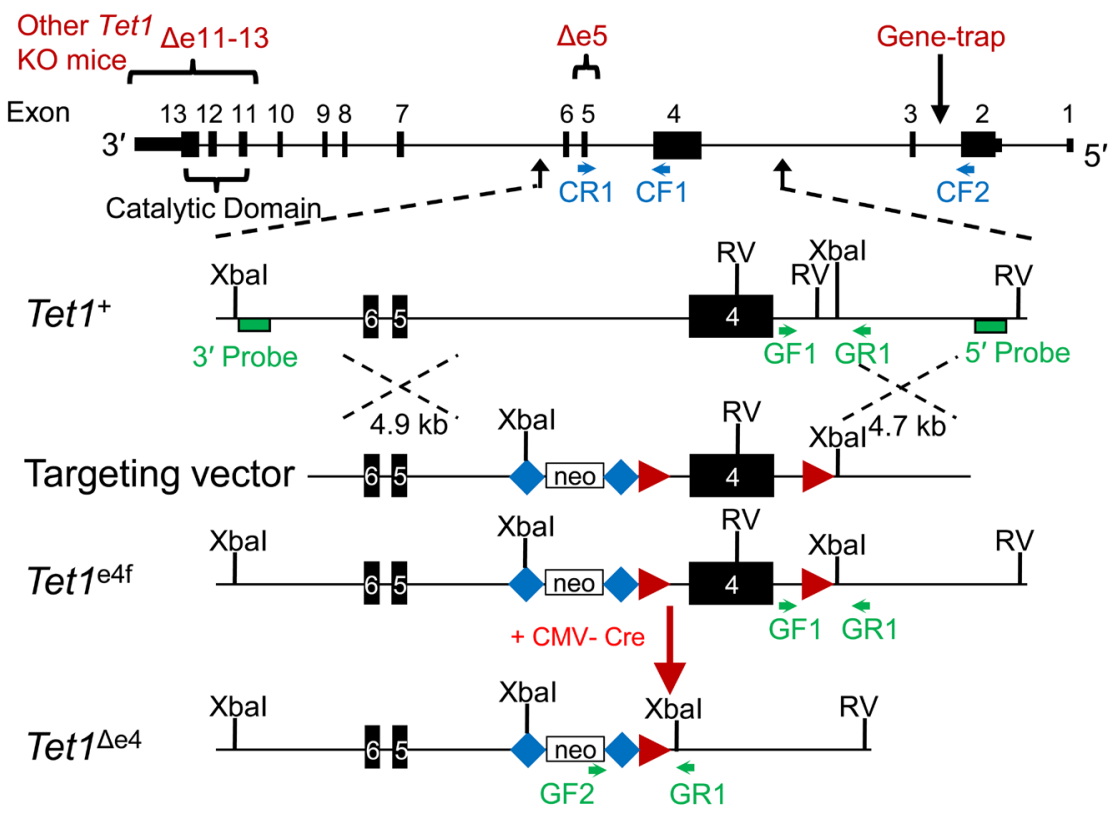

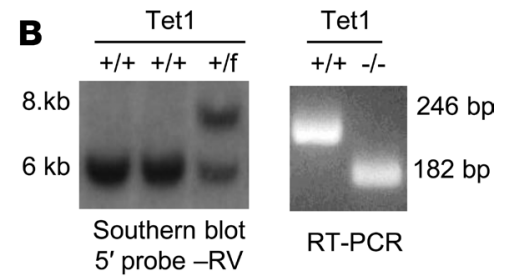

$\mathbf{C}$

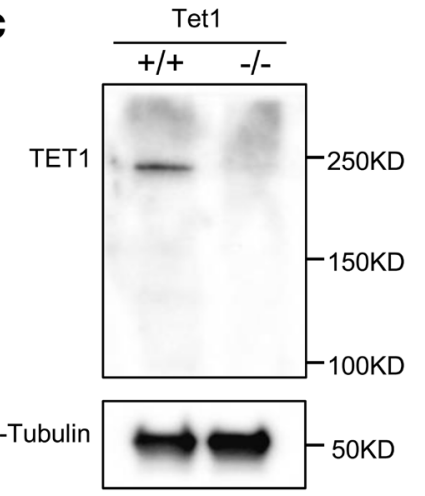

D

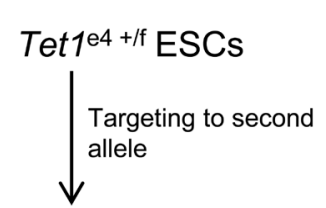

Tet ${ }^{\mathrm{e} 4 / \mathrm{f}} \mathrm{ESCs}$<smiles>[C+]1CC1</smiles>

Tet ${ }^{\Delta \mathrm{e} 4-\mathrm{I}-\mathrm{ESCs}}$

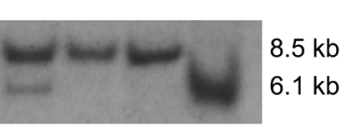

5' probe - EcoRV

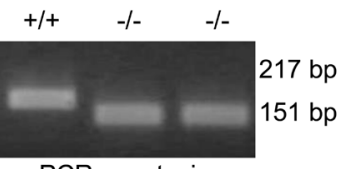

PCR genotyping
E

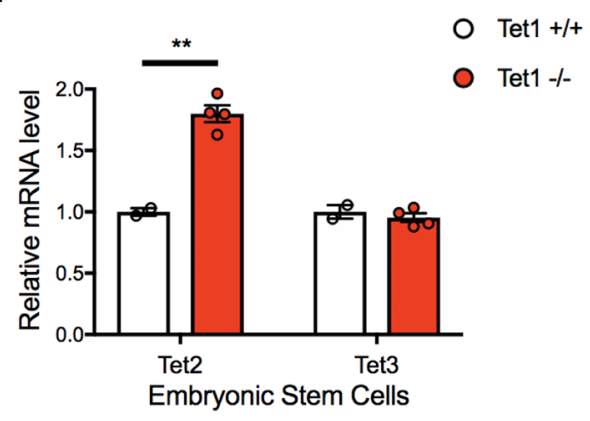

G

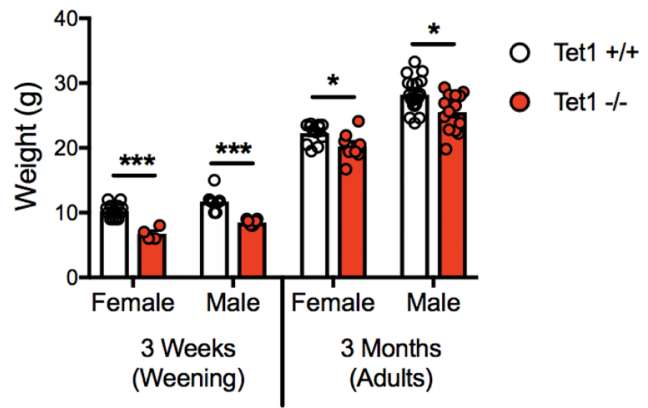

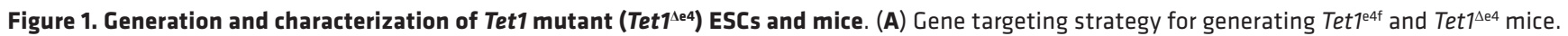
LoxP sites (red arrowheads), FRT sites (blue diamonds), and primers for RT-PCR (blue arrows) and genotyping (green arrows) are indicated. Mutations of other published Tet1 mutant mice are diagramed ( $\Delta \mathrm{e} 11-13, \Delta \mathrm{e}$, Gene-trap; refs. 42-44). (B) Left panel, DNA Southern blot confirmation of Tet1 ${ }^{\text {e4f }}$ (+/f) embryonic stem cells (ESCs). Right panel, RT-PCR of Tet1 $1^{\text {se- }- \text { - }}$ hippocampus. The primer pair of CR1 and CF1(246 bp) amplified the WT mRNA, and the primer pair of CR1 and CF2 (182 bp) detected the exon 4-deleted mutant mRNA. (C) Western blot analysis with a TET1 antibody confirmed deficiency of TET1 protein from Tet1 $1^{\text {1e4-/- }}(-/-)$ hippocampus. $\beta$-Tubulin used as loading control and fill uncut gel is shown. (D) Generation of homozygous Tet $7^{\text {Le- }-I-}$ ESCs through sequential targeting. The upper right panel shows the Southern blot confirmation of double-targeted ESCs ( $\left.f / f\right)$, and the lower right panel shows PCR genotyping confirmation of exon4 deletion (-/-) after electroporation with Cre plasmid (Supplemental Figure 1). (E) Tet2

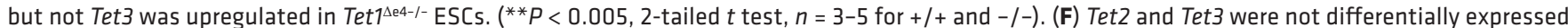

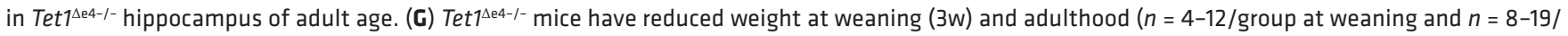
group at adult age). ${ }^{*} P<0.05 ;{ }^{* *} P<0.005,{ }^{* *} P<0.0005,2$-tailed $t$ test. All data are presented as mean $\pm \mathrm{SEM}$. 
Table 1. Tet1 pups (N6) surviving to weaning

\begin{tabular}{lccc}
\hline & $+/+$ & $+/-$ & $-/-$ \\
Observed & 79 & 128 & 41 \\
Expected & 62 & 124 & 62 \\
Genotype ratio at weaning. Fewer Tet1 & \\
\hline
\end{tabular}

We hypothesized that TET1-mediated accumulation of $5 \mathrm{hmC}$ is implicated in neuronal activity-regulated gene transcription in the brain. Tet 1 is expressed quite ubiquitously in the postnatal human and mouse brains with relative higher expression in the hippocampus (Supplemental Figure 2, A and B). Because neuronal activity-regulated gene transcription has been studied extensively in hippocampus (50), we then focused our studies in the hippocampus of Tet $1^{1 e 4-1-}$ mice using an electroconvulsive stimulation (ECS) paradigm to induce neuronal activity. We first validated the activity-dependent expression paradigm by confirming upregulation of Bdnf and Homerla after ECS (Supplemental Figure 3A) (51, 52). The effect of TET1 deletion on activity-dependent gene regulation was examined by comparing RNA sequencing (RNA-seq) expression profiles from the hippocampus of ECS-treated Tet $1^{\text {tet/-- }}$ and $\mathrm{Tet}^{\mathrm{T}^{+/}}$mice (Supplemental Figure 3B). The sequence coverage and depth are summarized in Supplemental Figure 3B. Examination of RNA-seq data reads mapped to Tet1 confirmed the deletion of exon 4 (Supplemental Figure 3C). Using a FDR $<0.05$ to compare the genes differentially expressed between Tet $1^{\text {1et-/-}}$ and Tet $1^{+/+}$mice, we discovered that 34 genes were significantly upregulated and 184 genes were downregulated (Figure 2A and Supplemental Table 1), suggesting a primary role for TET1 in activating gene expression. In order to test the hypothesis that TET1 is responsible for regulating activity-dependent genes, we cataloged a dataset of activity-dependent genes based on a literature review (Supplemental Table 2). While there was no significant enrichment for neuronal activity-regulated genes disrupted in Tet $1^{1 e 4-1-}$ mice in the RNA-seq data, dysregulation of several activity-dependent genes was identified, including the master memory gene regulator Npas4 (Figure 2B) (53). Npas4 downregulation was confirmed by quantitative PCR (qPCR) in the hippocampus of untreated Tet $1^{1 e 4-1-}$ mice, as well (Figure $2 \mathrm{C}$ ). A similar finding was previously reported in another line of Tet1 mutant mice (37). Gene Ontology (GO) analyses by DAVID of the dysregulated genes detected a significant enrichment for genes involved in the extracellular region/matrix/space (Figure 2D). In examining and verifying other nonactivity-dependent genes in RNA-seq data, we found significant downregulation of Oxtr, the transmembrane receptor that binds oxytocin and a key neuronal gene implicated in brain function and behavior. Oxtr downregulation was also observed in untreated Tet $1^{\text {te-l- }}$ mice but not in Tet $1^{1 e 4-/-}$ ESCs (Figure 2E).

We compared our list of Tet $1^{4 e-/-}$ dysregulated genes from ECS-treated hippocampus to the dysregulated genes reported in Tet1 exon 5-deficient $(\Delta \mathrm{e} 5)$ hippocampus $(37,43)$ and Tet1 exon $11-13$ deletion $(\Delta \mathrm{e} 11-13)$ neural progenitor cells (NPCs) (42). In Tet1 $\Delta \mathrm{e} 5$ mutant mice reported by Rudenko et al., a total of 258 genes are dysregulated in the hippocampus (37). A significant set of genes (54 genes, 20.9\%) was also found to be altered in the hippocampus of Tet $1^{\text {tet/- }}$ mice (Figure 2F). In NPCs of Tet1 $\Delta \mathrm{e} 11-13$ mice, only 4 of 54 genes (7.4\%) overlapped with the dysregulated genes in Tet $1^{\text {te4-/- }}$ mice. When we compared dysregulated genes from Tet1-knockdown ESCs by RNAi (47) or Tet1 Gt primordial germ cells (44), we did not find significant overlap with the dysregulated genes in the hippocampus of Tet $1^{\Delta e 4 /-}$ mice. These comparisons indicate TET1 differentially regulates sets of genes in a tissue-dependent manner. The differences between Tet 1 mutant mice may be due to the nature of the mutations disrupting specific isoforms of Tet1, as suggested by the alternative splicing of the Tet1 gene in a recent report (45).

DNA hypermethylation of the CpG island of Npas4 and Oxtr in Tet $1^{1 e 4-1-}$ mice. Based on the role of TET1 in DNA demethylation, we examined if the downregulation of Npas4 and Oxtr were associated with increased $\mathrm{CpG}$ island DNA methylation. We used targeted bisulfite genomic sequencing to examine the $\mathrm{CpG}$ island associated with the Npas4 promoter region and found it significantly hypermethylated in Tet $1^{1 e 4 /-}$ hippocampus $(27.2 \% \pm 3.1 \%)$ compared with $\operatorname{Tet}^{+/+}(8.1 \% \pm 0.6 \%)$ (Figure 3A and Supplemental Figure 4A). This is in line with previous data supporting a role for TET1 in regulating the methylation state of Npas4(37).

The mouse Oxtr gene contains 4 exons (6) including a 5' UTR, which encompasses exons 1, 2, and part of exon 3 and overlaps with an 859 bp CpG island (Figure 3B). The human CpG island spans 2,319 bp, 
A
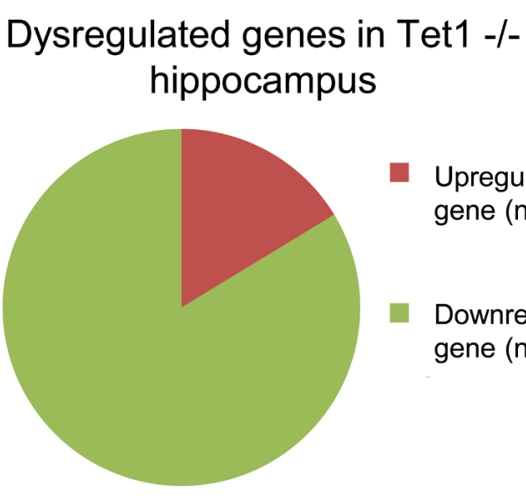

Upregulated gene $(n=37)$

Downregulated gene $(n=186)$

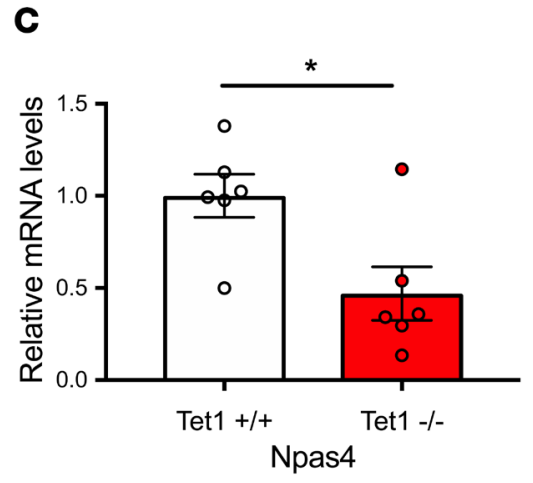

E

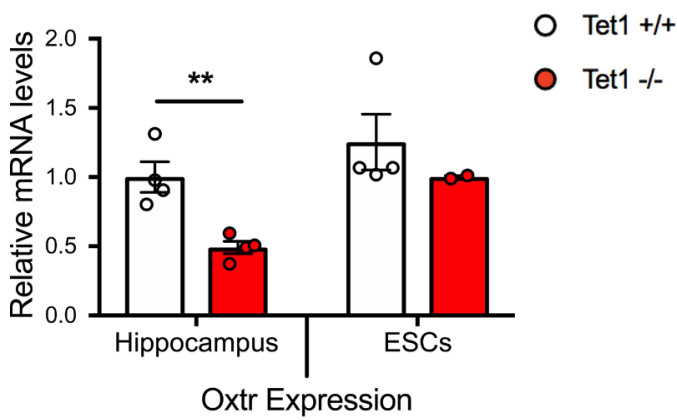

B

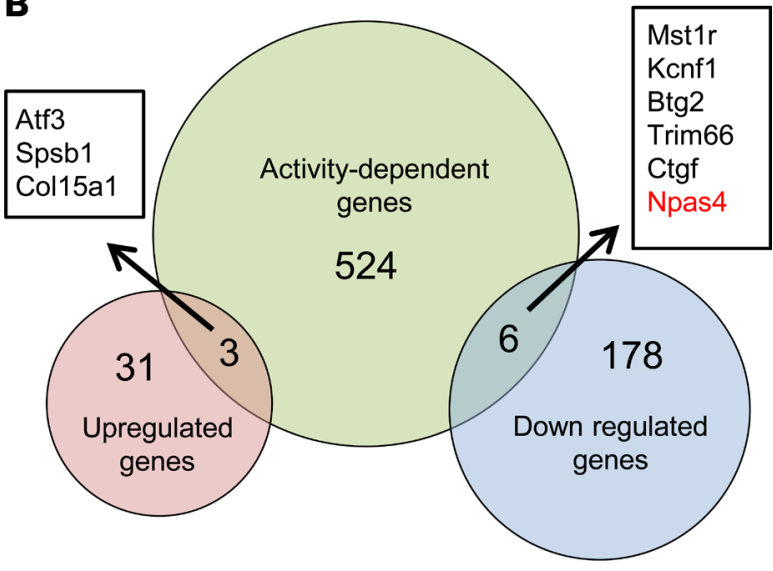

D Functional Annotation - GO

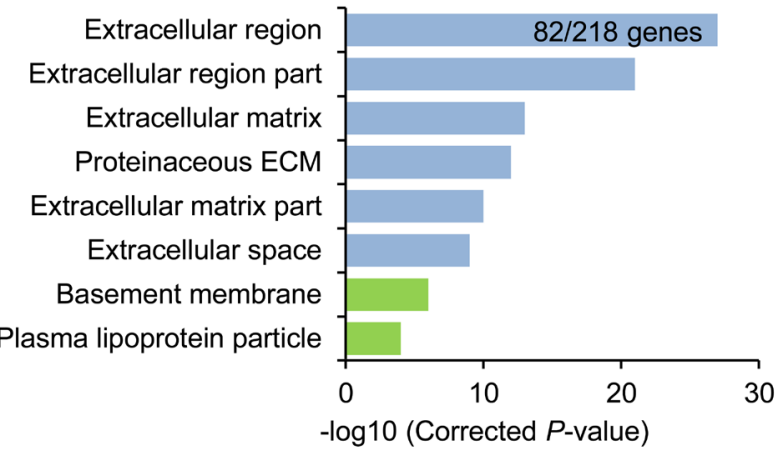

$\mathbf{F}$

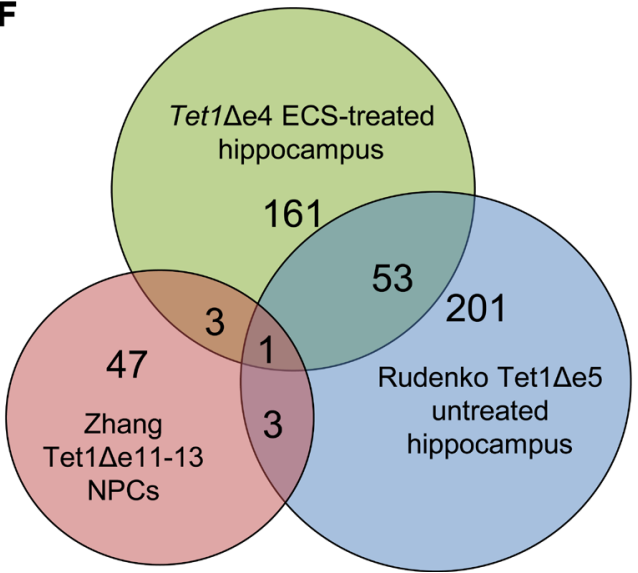

Figure 2. Tet1 deficiency results in significantly reduced expression of key neural genes, including Npas4 and Oxtr, in brain. (A) A total of 186 genes were downregulated and 34 were upregulated in Tet1 $1^{\mathrm{Ae} 4-\text { - }}$ hippocampus from RNA-seq analysis using FDR $<0.05$ ( $n=3 /$ group). (B) Overall, the dysregulated genes in Tet1 $1^{\text {Ae4-l- }}$ hippocampus did not have a significant overlap with known neuronal activity-regulated genes reported in literate (533 genes). Dysregulated activity-dependent genes such as Npas4 were identified from this analysis. (C) Npas4 was downregulated in hippocampus from Tet $1^{\Delta e 4-I-}$ mice $\left(n=6 /\right.$ group; ${ }^{*} P=0.017,2$-tailed $t$ test). (D) Gene Ontology (cellular component classification) analysis using DAVID revealed an enrichment of extracellular dysregulated genes in Tet $7^{\text {Ae4-I- }}$ mice (Benjamini corrected $P$ values indicated). (E) Oxtr was downregulated in hippocam-

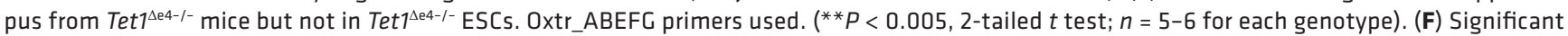
overlap of ECS-treated Tet $1^{\mathrm{\Lambda e4-I}-}$ hippocampal dysregulated genes with dysregulated genes from neural progenitor cells (NPCs) of Tet ${ }^{\mathrm{e} \text { e11-13 }}$ (odds ratio $[\mathrm{OR}]=7.66, P=0.003)$ and untreated hippocampus of Tet $7^{\mathrm{Se} 5}$ mice $\left(\mathrm{OR}=39.07, P=2.3 \times 10^{-57}\right)$. All data are presented as mean $\pm \mathrm{SEM}$. 
extending to the more $5^{\prime}$ noncoding region of OXTR (see detail in UCSC genome browser for both mouse and human OXTR/Oxtr CpG islands; www.genome.ucsc.edu). The mouse Oxtr gene also has high GC-content in the homologous region to the human promoter $\mathrm{CpG}$ island, indicating that the difference in $\mathrm{CpG}$ island length is likely due to a difference in computational thresholds rather than a biological difference. The CpG islands in both human and mice are located downstream of the characterized canonical promoter $(5,6)$ (Figure $3 \mathrm{~B}$ ). This suggests the presence of additional regulatory elements, such as an additional promoter within the $\mathrm{CpG}$ island. In silico analysis of ENCODE data also revealed histone modification peaks within the $\mathrm{CpG}$ island that support the presence of regulator elements for OXTR. To examine if the reduced hippocampal expression of Oxtr was associated with DNA hypermethylation of potential regulatory elements within the Oxtr gene, methylation in the $5^{\prime} \mathrm{CpG}$ island and adjacent region (BS1-BS3) of hippocampus of adult mice was analyzed by targeted bisulfite sequencing (Figure 3B). We first performed bisulfite sequencing on BS1, a region mostly upstream of the 5' Oxtr transcription start site (TSS). Overall, we were able to achieve $>99.0 \%$ of the C-T bisulfite conversion rate for most experiments, and only the sequence products that reached $>99 \% \mathrm{C}$-T conversion rate were included for data analysis. Consistent with the association of low DNA methylation at transcriptionally active promoter regions, BS1 is mostly unmethylated and comparable between $\operatorname{Tet}^{+/+}(8.45 \% \pm 0.51 \%)$ and Tet $1^{\text {tet-/- }}(10.13 \% \pm 0.66 \%)$ mice, indicating that methylation in the BS1 region is probably not responsible for the reduced expression of Oxtr (Figure 3, B and D, and Supplemental Figure 4B). We next examined BS2, a region just downstream of the most $5^{\prime}$ TSS. A portion of this region has been shown in humans to have activity for an alternative promoter that is suppressed by DNA methylation (region MT2 in Figure 3B) (9). Tet $1^{\text {tet-/- }}$ mice have significantly increased DNA methylation $(9.55 \% \pm 1.48 \%)$ in BS2 compared with their Tet $1^{+/+}$littermates $(2.62 \% \pm 0.61 \%)(P=$ 0.0015 ) (Figure 3, B and D, and Supplemental Figure 4B). The differential methylation in the BS2 region appears to be driven by only a few $\mathrm{CpG}$ dinucleotides, including the $\mathrm{CpG}$ sites that may be homologous to the human CpG -934 (The "A" in the ATG codon is denoted as +1 position) which was hypermethylated in a small sample of autistic individuals (14) and correlates with several morbid behaviors (54-56). ChIP data from the ENCODE project (57) indicate that the CpG island spanning Oxtr exon 3 includes a potential regulatory element. Strikingly, nearly every $\mathrm{CpG}$ assessed in this region (BS3) is significantly hypermethylated in Tet $1^{1 e 4-/-}$ mice $(70.6 \% \pm 3.60 \%)$ compared with their $T e t 1^{+/+}$littermates $(22.03 \% \pm 3.23 \%)(\mathrm{p}<0.0001)$ (Figure 3, B and D, and Supplemental Figure 4B). To test for a gene dosage effect of TET1 on Oxtr DNA methylation, we examined the BS3 region in heterozygous Tet $1^{4 e 4+/-}$ mice and found an intermediate level of methylation $(42 \% \pm 3.3 \%)$ that is significantly lower than Tet $1^{1 e 4-/-}(n=4, P=0.0019)$ (Figure 3, B and D, and Supplemental Figure 4B). Because bisulfite genomic sequencing method cannot distinguish between $5 \mathrm{mC}$ and $5 \mathrm{hmC}$, we performed hydroxymethylated DNA immunoprecipitations (hMeDIP) using an antibody specific for $5 \mathrm{hmC}$ for the BS3 region, and we conducted qPCR on immunoprecipitated DNA. This analysis did not reveal any dignificant difference in $5 \mathrm{hmC}$ abundance in the BS3 region between Tet $1^{-/-}$and Tet $1^{+++}$animals. This result further supports that the difference in methylation detected by bisulfite sequencing is due to a difference in $5 \mathrm{mC}$, not $5 \mathrm{hmC}$ (Figure 3C).

We examined the level of BS3 methylation in ESCs to test if the correlation between Oxtr gene expression and DNA methylation extended to ESCs. Consistent with our findings that Oxtr was not differentially expressed in ESCs, BS3 methylation in Tet1 $1^{\text {tet/- }}$ ESCs is not different from Tet1 $1^{+/+}$ESCs (Figure 3E). These data suggest that DNA methylation of BS3 regions is associated with altered Oxtr expression, as ESCs do not express Oxtr. To examine brain region specificity of Oxtr $\mathrm{CpG}$ island hypermethylation, we examined multiple brain regions and found the hypermethylation of BS2 and BS3 in Tet $1^{1 e 4 /-}$ adult mouse brain is not limited to hippocampus but also includes the regions of cerebellum, cerebral cortex, and olfactory bulb (Figure $3 \mathrm{~F}$ and Supplemental Figure 4C). These results suggest that hypermenthylation of Oxtr in Tet $1^{4 e 4 /-}$ animals may be established early in brain development prior to the development of different brain regions. We examined BS3 in embryonic brains and found hypermethylation of Oxtr in the cerebrum as early as E14.5 (Figure 3G). As it is technically challenging to genotype and analyze the DNA methylation patterns of each germ layer of an individual embryo due to an insufficient amount of DNA, we speculated that if hypermethylation of BS3 was established and maintained prior to gastrulation, we would find adult tissues from all 3 primary germ layers to be hypermethylated. In addition to our finding of hypermethylation in the adult brain (ectoderm), we observed BS3 hypermethylation in tissue that arose from mesoderm (heart) and endoderm (lung) in adult Tet $1^{1 e 4-1}$ mice (Figure 3H). As BS3 methylation is similar in ESCs between WT and Tet $1^{1 e 4 /-}$, these data suggest a window during early embryonic development for TET1-mediated maintenance of Oxtr methylation, which is TET1-dosage dependent. 
A

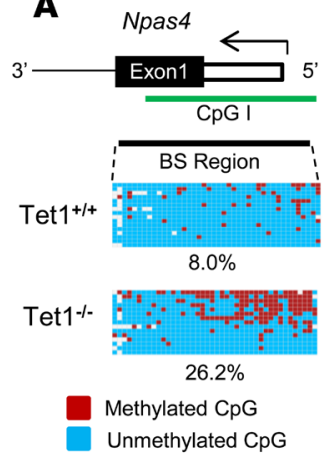

B

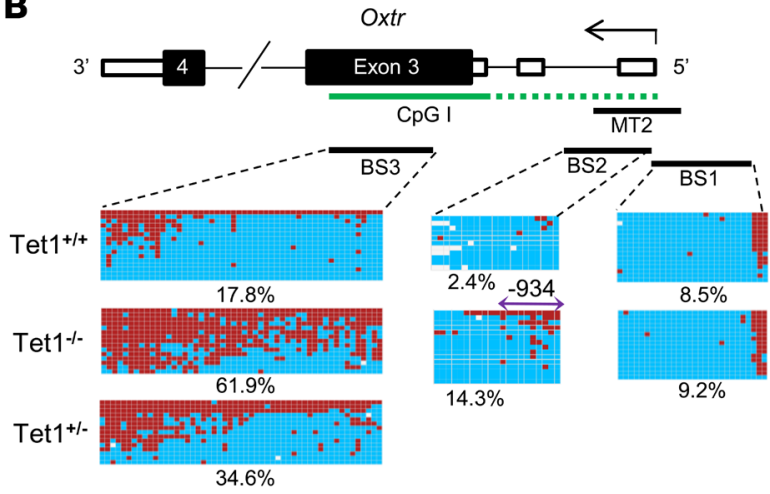

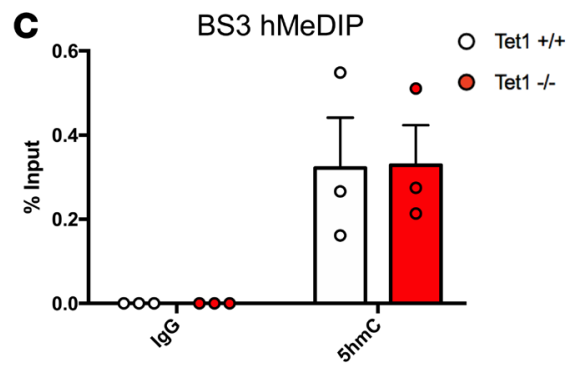

D
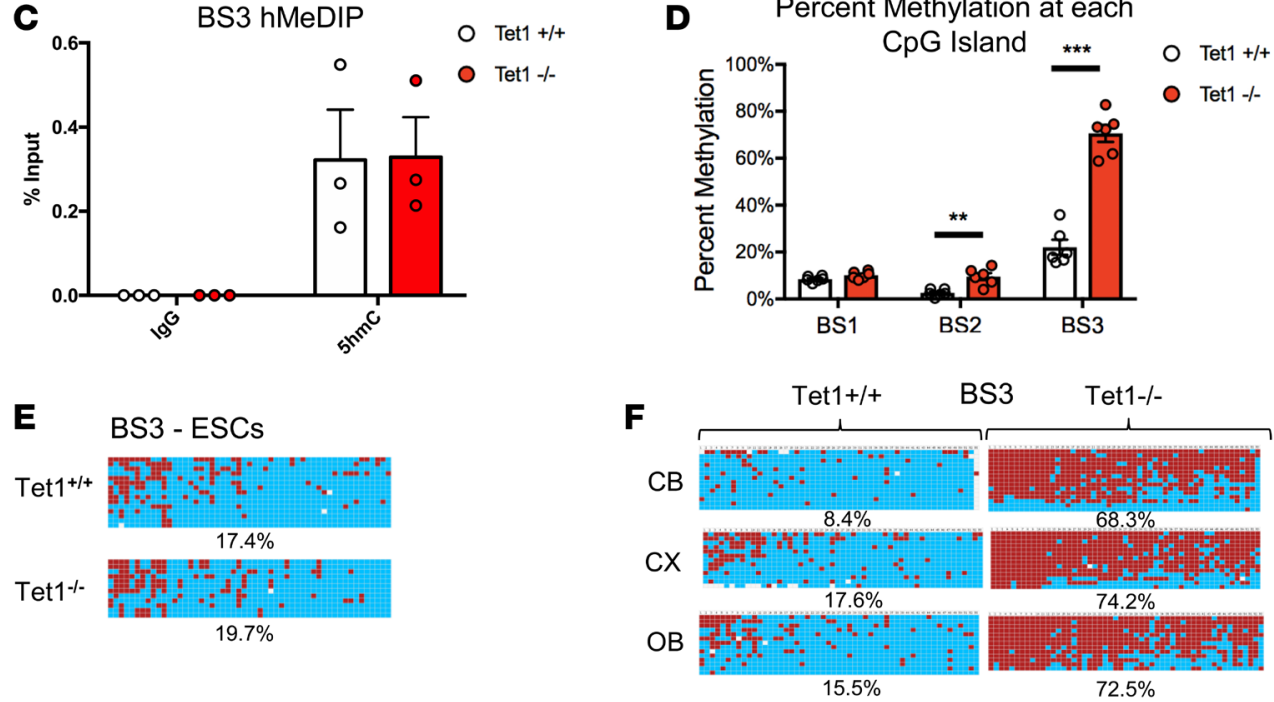

G BS3 - E14. 5 cerebrum

I

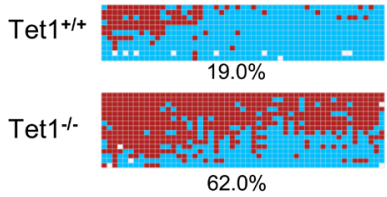

H BS3 - Heart (Meso)

BS3 - Lung (Endo)
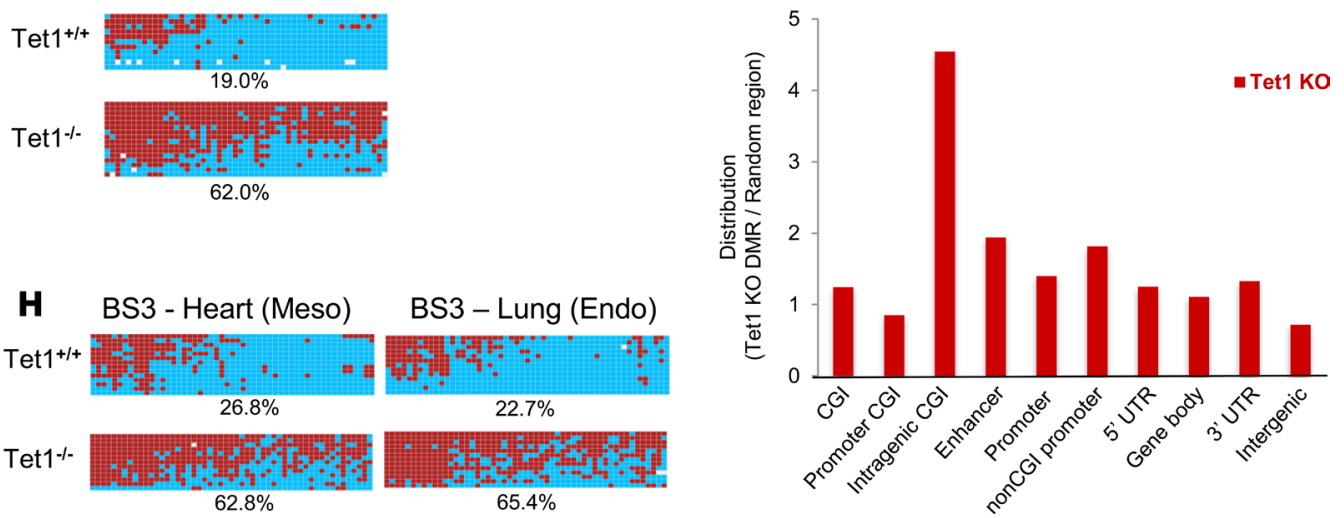

Figure 3. Tet1 1e4-I- mice show hypermethylation of the Npas4 and Oxtr $\mathrm{CpG}$ islands during early development and complex transcriptional dysregulation of Oxtr. (A) Diagram of Npas4 promoter (coding regions are shaded), associated CpC island (green bar), and bisulfite-sequencing region (black bar). Npas 4 was hypermethylated in hippocampus of Tet $7^{\mathrm{Ae} 4-1-}$ mice $(n=3 /$ group; $P=0.004$, 2-tailed $t$ test). Blue squares represent unmethylated CpC dinucleotides, red squares represent methylated $\mathrm{CpGs}$, and white squares were undetermined due to the ambivalent sequence reads and the same for other figures. (B) Diagram of Oxtr gene structure (coding regions are shaded), CpG island (green bar), and bisulfite-sequencing regions (BS, black bars). The genomic coordinate of BS1-BS3 in mouse mm9 assembly are as follows: BS1, Chr6:112440814-112441327; BS2, Chr6:112440387-112440815; BS3, Chr6: 112439019-112439542. The human CpG island spans 2319 bp (hg19:Chr3:8808962-8811280) extending to the more $5^{\prime}$ region of OXTR as indicated by a dotted green line. Mouse CpG island is 859 bp (mm9:Chr6:112439019-112439877). Human promoter MT2 region (9) and the region harboring the CG site that is likely to be equivalent to the human -934 CG site (14) are indicated as arrow. BS2 and BS3 were hypermethylated but not BS1 in hippocampus of adult Tet $7^{\mathrm{e} 4-/-}$ mice ( $n=3 /$ group; BS2, $P=0.0015$; BS3, $P=0.0000015$; 2-tailed $t$ test). BS3 showed intermediate levels of hypermethylation in Tet $7^{\mathrm{Ae} 4+/-}$ mice $(n=$

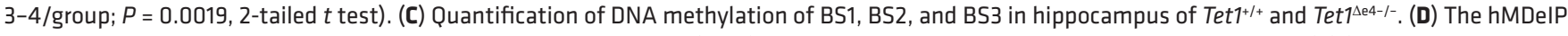

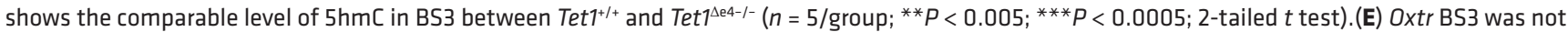

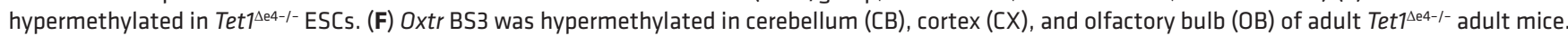
(C) Oxtr BS3 was hypermethylated in E14.5 cerebellum of Tet 1 $^{\mathrm{e} 4-I-}$ mice. (H) Oxtr BS3 was hypermethylated in tissues of heart and lung from the other 2 germ layers (Meso, mesoderm; Endo, endoderm). (I) Whole-genome bisulfite sequencing of neocortex from adult brain of Tet ${ }^{\text {1e4-I- }}$ mice revealed Tet1DMRs are significantly enriched in intragenic CpC islands (CGI) ( $n=3$ for $+/+$ and $-/-; P=2.65 \times 10^{-38}$, Fisher's exact test). 
The TET1 protein contains a CXXC domain, which preferentially binds to unmethylated CpG-rich sequences (58). We performed whole-genome bisulfite sequencing in adult cerebral cortex tissue using a previously described method $(45,59)$ to investigate the genome-wide effect of TET1 deficiency on DNA methylation in the brain. As whole genome bisulfite sequencing requires larger quantities of input DNA, cerebral cortex tissue was chosen because the size of the tissue allows for a sufficient amount of DNA to be obtained from a single animal. Sequence reads were mapped to mouse genome mm9 assembly (http:// genome.ucsc.edu) using BSMAP program (60), and only samples with $\mathrm{C}$ to $\mathrm{T}$ conversion rate greater than 99\% were accepted for further data analysis. Through this analysis, we identified 666 differentially methylated regions (Tet1-DMRs) between Tet $1^{\text {set-/- }}$ and $T e t 1^{1 /+}$, with an enrichment for hypermethylated Tet1DMRs (522 hypermethylated vs. 144 hypomethylated). We discovered an enrichment of hypermethylated Tet1-DMRs in CpG islands (380 CpG islands; $\triangle \mathrm{mCG} \geq 20 \%$ ), including the Oxtr CpG island (1.2 fold observed/random, $P=1.25 \times 10^{-7}$ ) (Supplemental Table 3 ). The distributions for DMRs indicated a distinct pattern across the genome. Stratifying $\mathrm{CpG}$ islands by location (promoter vs intragenic) revealed Tet1DMRs significantly enriched in intragenic CpG islands (Figure 3I, 4.5-fold observed/random, $P=2.65 \times$ $10^{-38}$ ), indicating a more prominent role for TET1 in regulating intragenic $\mathrm{CpG}$ island methylation. The motif analysis by HOMER2 program has revealed top 20 binding sites including NeuroD and MEF2C, which are known to be important for transcriptional regulation of neuronal genes (46) (Supplemental Figure $5 \mathrm{~B}$ ). In addition, the methylation at the $\mathrm{mCH}$ site is not significantly different between $\mathrm{Tet}^{+/+}$and Tet $1^{4 e 4-1-}$ mice (Supplemental Figure 5A).

Discovery of Oxtr gene mRNA isoforms and reduced expression of Oxtr Isoforms in Tet $1^{\text {te4-l- }}$ mice. The pattern of Oxtr hypermethylation within BS3 and not BS1 suggests that a regulatory element lies within exon 3 of Oxtr, which could cause Oxtr to have a more complex transcript structure than described in the literature $(5,6,61)$. A single $O x t r$ transcript (Oxtr-A) has been previously characterized with an ORF spanning exons 3 and 4 and encodes a 388-amino acid protein (6) (Figure 4A). Examining the Oxtr gene structure in the genome browser (mm9, http://genome.ucsc.edu/) revealed a predicted mRNA isoform lacking exons 1 and $2(O x t r-B)$, as well as a previously deposited isoform originating from intron $3(O x t r-C)$. We validated the presence of all 3 isoforms in the hippocampus by RT-PCR and sequencing (Figure 4A). In addition, we identified a fourth isoform by RT-PCR and sequencing in which exon 3 is skipped $(O x t r-D)$. To examine if there were additional TSS not previously reported that could be affected by the exonic hypermethylation of the BS3 region, we performed 5' rapid amplification of cDNA ends (RACE) using primers from exon 4 in a pair of $\mathrm{Tet}^{+/+}$and Tet1 $1^{1 e 4-/-}$ mice. Interestingly, the sequences of $5^{\prime}$ RACE products revealed 4 potentially novel TSS, including 1 originating from intron $3(O x \operatorname{tr} E-H)$ (Figure $4 \mathrm{~A})$. Isoforms $\mathrm{A}$ and $\mathrm{B}$ have the same full-length ORF, while the other mRNA isoforms have predicted shorter ORFs ranging from 75-114 amino acids. However, whether the shorter mRNA isoforms are translated endogenously and possess a function similar to OXTR warrants further investigation. We measured the relative abundance of these various $O x t r$ transcripts by qPCR with primer sets indicated in Figure 4A. Our results indicate that $O x t r-B$ is the most dramatically reduced isoform ( $20 \%$ of WT), while full-length $O x t r-A$ and potentially novel isoform $O x t r-H$ are not different in Tet $1^{1 e 4-/}$ hippocampus (Figure 4B). Transcript levels of isoforms $\mathrm{C}$ and $\mathrm{D}$ were below our detection threshold, and unique primers to detect isoforms E, F, and G could not be designed because of transcript overlap. Our data together indicate a complex transcriptional regulation with the possibility of more than 1 TSS of Oxtr. This data also suggests a mechanism of TET1-governed DNA methylation underlying expression of selective isoforms of Oxtr.

The histone modifications are altered in DMRs of Oxtr in Tet $1^{1 e 4-/-}$ mice. DNA methylation is mechanistically known to be linked to various histone modifications in the genome (62). DNA hypermethylation of BS3 and reduced transcripts of Oxtr in Tet $1^{1 e 4 /-}$ mice prompted us to examine whether histone modifications associated with Oxtr are altered. We first examined the ChIP-seq data of mouse brain in the Oxtr region from the ENCODE project (http://genome.ucsc.edu) (63). Consistent accumulation of both the active histone mark $\mathrm{H} 3 \mathrm{~K} 4 \mathrm{me} 3$ and the repressive mark H3K27me3 suggest that a bivalent peak of histone modifications may also contribute to expression of Oxtr in various tissues (Figure 4C and Supplemental Figure 6). For example, the peaks of H3K4me3 ChIP-seq data in heart tissue show a distinct pattern at E14.5 and 8-week-old mice during development (Supplemental Figure 6). The bivalent chromatin regions overlap with the canonical TSS and the hypermethylated BS3/BS2 region within CpG island in Tet1 mutant mice. These in silico data strongly suggest additional transcriptional regulatory elements in BS3/BS2 hypermethylated that are associated with different histone modifications. 
A

Oxtr isoform A (D86559; 388 aa)

Oxtr isoform B

Oxtr isoform C (KU686796; 75 aa)

Oxtr isoform D (KU686797;75 aa)

Oxtr isoform $\mathrm{E}$ (KU686798;114 aa)

Oxtr isoform $\mathrm{F}$ (KU686800;114 aa)

Oxtr isoform $\mathrm{G}$ (KU686800;114 aa)

Oxtr isoform $\mathrm{H}$ (KU686800;114 aa)

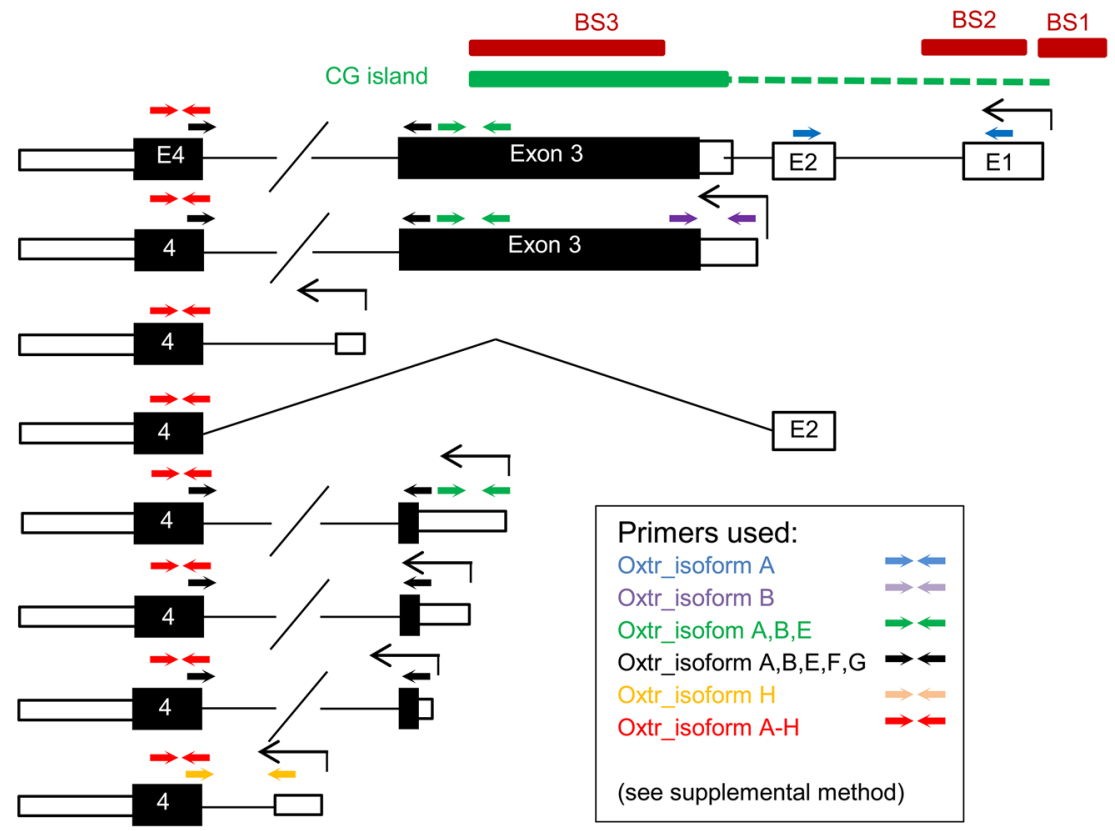

B

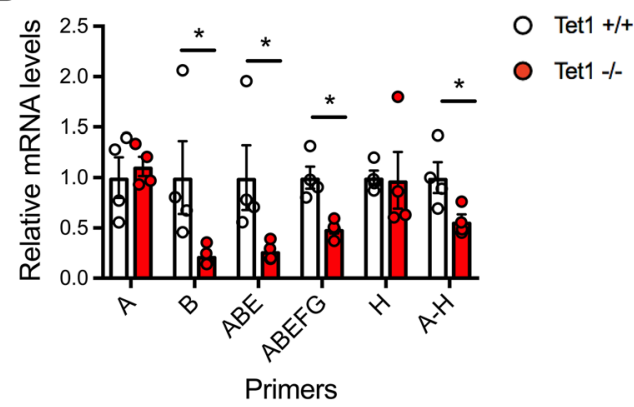

C

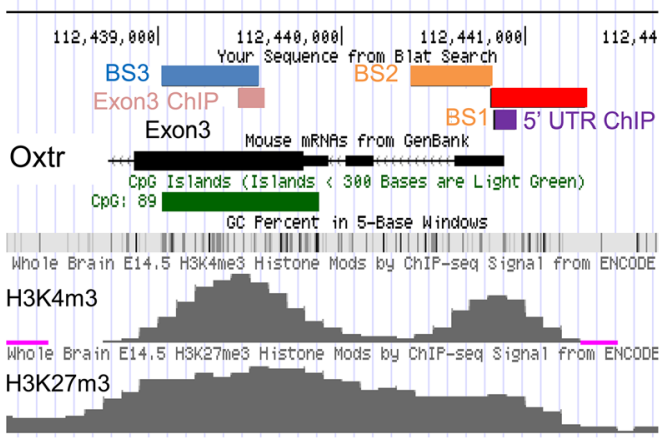

\section{D}

Histone modification in Tet1 DMR (2kb bin)

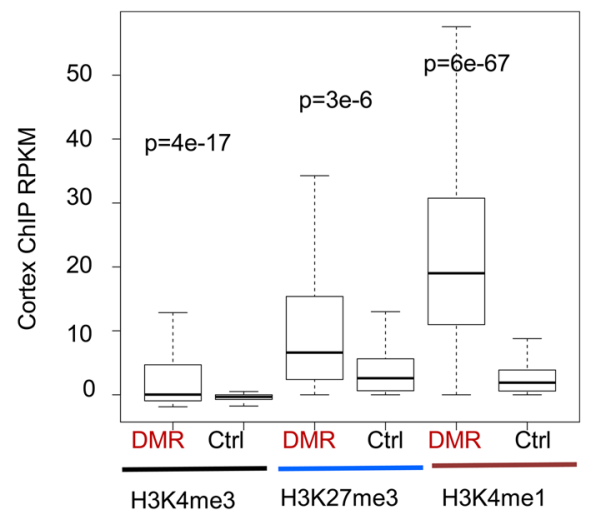

E

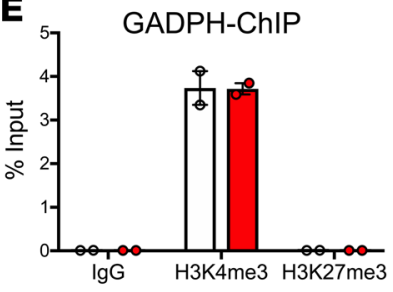

$\mathbf{F}$

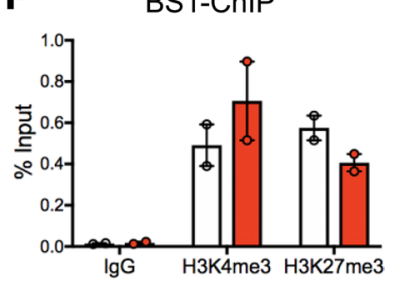

G

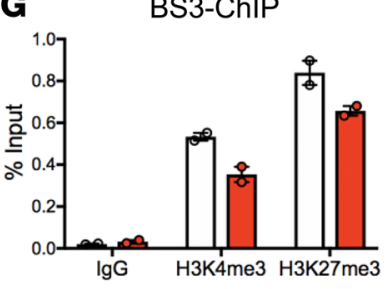

Figure 4. Identification of Oxtr mRNA isoforms and reduced expression of Oxtr isoforms in Tet1 ${ }^{\text {ee4-I- }}$ mice. (A) Oxtr mRNA isoforms (A-H) identified by $5^{\prime}$ RACE and confirmed by RT-PCR and Sanger sequencing (predicted coding regions are shaded). qPCR primers indicated (multicolored arrows and supplemental method). The new sequences for individual isoforms have been deposited in GeneBank with accession no. KU686795-KU686801. (B) qPCR data revealed downregulation of isoform $\mathrm{B}$, but not $\mathrm{A}$ or $\mathrm{H}$, in the hippocampus of Tet $1^{\mathrm{Ae4-}-\mathrm{-}}$ mice $\left(n=3-4 / \mathrm{group},{ }^{*} P<0.05,2\right.$-tailed $t$ test). (C) The histone modification of Oxtr in mouse brain. The enrichment of histone H3K4me3 and H3K27me3 in BS1-BS3 regions of Oxtr was revealed from ENCODE project. A second putative regulatory element was identified within exon 3 (large black bar) of Oxtr, which overlaps a CpG island (green bar) (mm9, http://genome.ucsc.edu/). Selection of Oxtr regions for bisulfite sequencing (BS1-BS3) and ChIP-PCR are indicated. ENCODE ChIP data shown is from E14.5 whole brain produced in the laboratory of Bing Ren (H3K4me3, GEO GSM1000095; H3K27me3, GEO GSM1000143). The similar pattern is also observed in ChIP-seq from 8-week cerebellum 
(http://genome.ucsc.edu) (Supplemental Figure 6). (D) The enrichment of H3K4me3, H3K27me3, and H3K4me1 in DMRs of Tet7 194-/- mice. The hypermethylated DMRs (200 bp bin) in Tet1 ${ }^{\Delta e 4-I-}$ cortex was selected firstly by Fisher test with a threshold of $P<0.05$. The 2000 bp genomic windows containing at least 4 hypermethylated DMRs (200 bp) in Tet $7^{\text {se4-/- }}$ cortex were selected out as DMRs for histone enrichment analysis. Random control regions with same bin size covered by the same methylome were chosen as controls. The ENCODE ChIP-seq data from WT adult mouse neocortex was used for the analysis. $P$ values (calculated by $t$ test) indicate a significant differential histone enrichment between DMRs and control region. (E-G) ChIP-qPCR revealed altered histone modifications at the bivalent promoter region of and GARDP control (E) and Oxtr (F and $\mathbf{G}$ ) in the cerebrum of Tet $7^{\text {(e4-I- }}$ mice. H3K4me3 (active mark), H3K27me3 (repressive mark), and IgG (isotype negative control) were assessed in 2 regions overlapping coding exon 3 and exon 1 in the 5' UTR. Both H3K4me4 and H3K27me3 were reduced in BS3 hypermethylated region of Tet ${ }^{1 \mathrm{e4}-\text { - }}$ mice $(n=2 /$ group and 3 replicates for each group).

We then compared the histone modifications of $\mathrm{H} 3 \mathrm{~K} 4 \mathrm{me} 3, \mathrm{H} 3 \mathrm{~K} 27 \mathrm{me} 3$, and $\mathrm{H} 3 \mathrm{~K} 4 \mathrm{me} 1$ between the DMRs identified in the cerebral cortex of Tet $1^{\text {tet-1- }}$ mice and randomly selected $2 \mathrm{~kb}$ bin control genomic regions genome-wide from the same methylome using ChIP-seq data in mouse cortex deposited by ENCODE (63). This analysis revealed that the modifications of H3K4me3, H3K27me3, and H3K4me1 were enriched in the DMRs of Tet $1^{\text {teq-/- }}$ mice compared with the control regions (Figure 4D). Accordingly, ENCODE ChIP-seq data from E14.5 whole brain tissue indicates the Oxtr promoter has 2 active H3K4me3 peaks and a repressive H3K27me3 peak that overlap the BS1-3 regions (Figure 4C), typical of a bivalent domain (64). TET1 is known to preferentially bind to these transcriptionally poised bivalent domains, which are generally hypomethylated in ESCs (47). To examine whether the histone modifications of $\mathrm{H} 3 \mathrm{~K} 4 \mathrm{me} 3$ and $\mathrm{H} 3 \mathrm{~K} 27 \mathrm{me} 3$ suggested from in silico analysis were altered in Tet $1^{\text {tes-I- }}$ mice, we performed ChIP analysis using hippocampus tissues and GADPH as control to show the specificity of the antibody (Figure 4E). H3K4me3 was increased and H3K27me3 was not changed in the BS1 region (Figure $4 \mathrm{~F}$ ). $\mathrm{H} 3 \mathrm{~K} 4 \mathrm{me} 3$ and $\mathrm{H} 3 \mathrm{~K} 27 \mathrm{me} 3$ were reduced in the hypermethylated BS3/ exon 3 region in Tet $1^{4 e 4-1}$ mice (Figure 4G). The degree of reduction of H3K4me3 (active chromatin mark) was more prominent than that of $\mathrm{H} 3 \mathrm{~K} 27 \mathrm{me} 3$ (repressive chromatin mark). Hypermethylation of Oxtr skews the balance between the active and repressive marks, resulting in abundance of H3K27me 3 and the reduction of select mRNA isoforms of Oxtr.

Tet $1^{\text {1e4-I- }}$ mice display abnormal social behaviors. Due to the observed dysregulation of Npas4 and Oxtr and their roles in synaptic development and function $(65,66)$, we wanted to test whether there would be cognitive and social deficits in Tet $1^{1 e t-1-}$ mice. We performed a battery of behavioral tests using multiple cohorts of backcrossed (N6) C57BL/6J mice (Supplemental Figure 7). Tet1 $1^{\text {set-1- }}$ mice appeared grossly normal by a neurophysiological screen (Supplemental Figure 8). Tet $1^{\text {set-/- }}$ mice were hypoactive and spent less time in the center of the open field (Figure 5A and Supplemental Figure 9A) but showed an enhanced ability to stay on the accelerating rotarod (Supplemental Figure 9B), suggesting the hypoactivity in the open field is more likely anxiety related than a motor impairment. However, Tet $1^{\text {te4-1- }}$ mice do not have a strong anxiety phenotype in the light-dark emergence test (Supplemental Figure 9C).

The role of OXTR has been well studied in the context of social and maternal care behaviors $(66,67)$, and increased aggression and reduced maternal care are observed in Oxtr mutant mice (68). To examine whether Tet $1^{\text {te-l- }}$ mice show similar abnormal behaviors, we first performed the resident intruder test. In the sex-matched resident intruder test, there was a significant increase in agonistic behaviors in female Tet $1^{\text {set-1- }}$ mice but not in male Tet $1^{1 e 4-/}$ mice (Figure 5B and Supplemental Figure 9D). Tet $1^{\text {tet/- }}$ female mice display threatening postures of a similar magnitude to male mice. These abnormal behaviors are similar to that observed in $\mathrm{Oxtr}^{-1}$ mice (68). We further evaluated the maternal care behavior by virgin pup-retrieval test. Maternal care behavior was only tested in virgin females, as Tet $1^{1 e 4-1-}$ female mice have reduced fertility and could not produce enough pups for dam pup retrieval. Increased maternal responsiveness after repeated exposures to pups is associated with increase oxytocin expression in the brain of WT virgin female mice (69, 70). Interestingly, the virgin pup retrieval test revealed that Tet $1^{1 e 4-1}$ female mice show increased latency to retrieve pups on the third day of testing (Figure 5C, left panel), spend less time crouching over pups (Figure $5 \mathrm{C}$, middle panel), and show an increase in aggressive interactions of pup exposure (Figure 5C, right panel). Male mice were not tested in virgin pup retrieval, as males are known to attack nonoffspring (71).

In the potentially novel object recognition test, Tet $1^{\text {set-/ }}$ mice show a trend toward short-term (1 hour) memory deficits, indicating impairment in episodic memory (Figure 5D). Long-term (24 hours) memory is not significantly different between genotypes. Spatial learning (Morris water maze) (Supplemental Figure 9, E and F), olfactory memory (social transmission of food preference) (Supplemental Figure 9G), and associative memory (fear conditioning) did not differ between Tet ${ }^{\text {ted-1- }}$ mice and their Tet $1^{+/+}$littermates (Supplemental Figure 9H). 
A

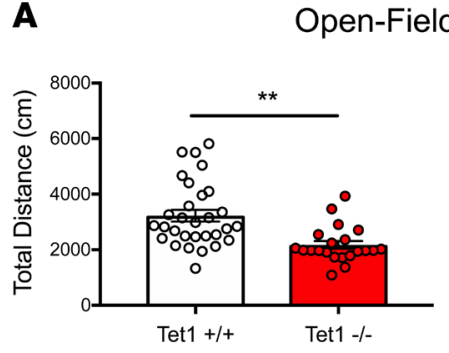

C Latency to $1^{\text {st }}$ Retrieval

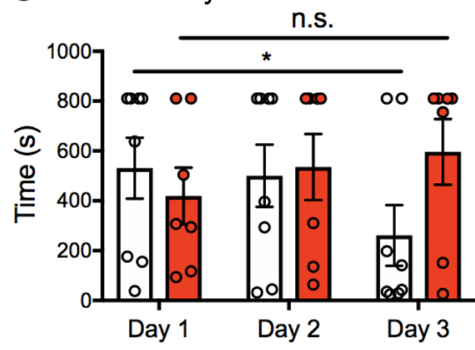

D Novel Object Recognition
B Resident-Intruder

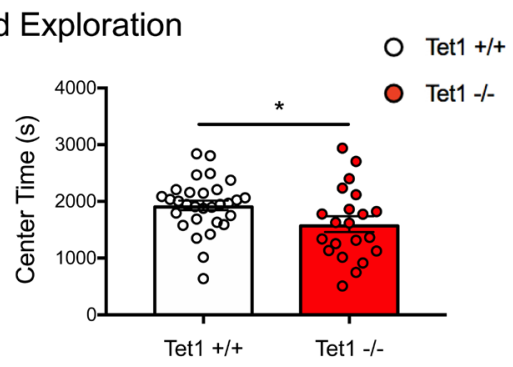

Crouching Duration
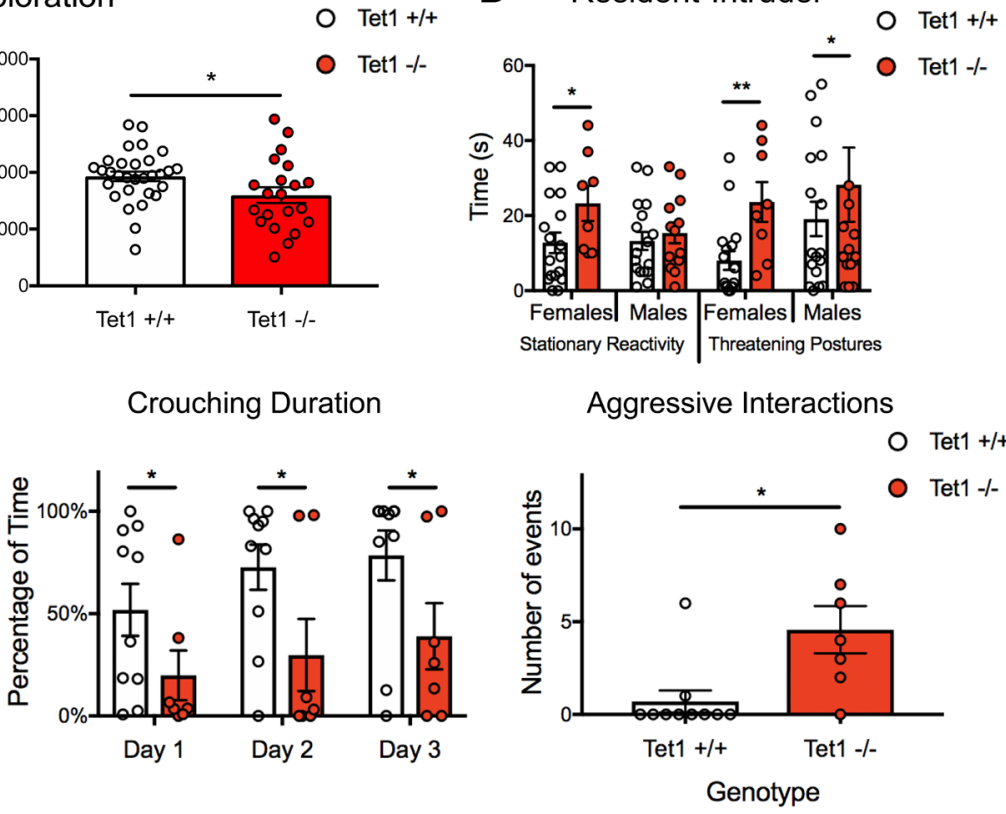

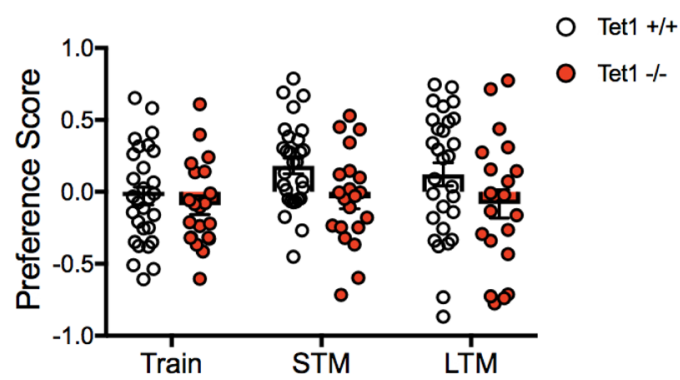

Figure 5. Tet $1^{\text {Ae4-/- }}$ mice are hypoactive and display impaired social and maternal care behaviors. (A) Tet $7^{\text {se4-/- }}$ mice were hypoactive in the open-field exploration as indicated by a reduced distance traveled $(P=0.0005,2$-tailed $t$ test $)$ and reduced center time $(P=0.04,2$-tailed $t$ test $) . n=21(-/-)$ and 30(+/+). (B) Tet $7^{1 \mathrm{e} 4-/-}$ female mice display significantly increased stationary reactivity $(P=0.017,2$-tailed $t$ test) and threatening postures in the resident intruder test $(P=0.001,2$-tailed $t$ test). Males show a significant increase in threatening postures $(P=0.02,2$-tailed $t$ test). $n=13$ (male- $/-)$ and 8 (female-/-); $n=17$ (male $+/+$ ) and 13 (female+/+). (C) Tet $1^{\text {Le4-I- }}$ mice showed delay in pup retrieval (genotype $x$ day: $F[2,30]=7.244, P=0.003$, Tukey's

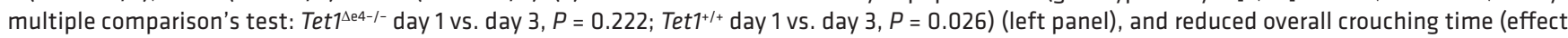
of genotype: $F[1,15]=5.357, P=0.035$ ) (middle panel). Both genotypes show an increase in crouching over time (effect of day: $F[2,30]=3.534$,

$P=0.042)$; however, post-hoc analysis were not significant for any group. $n=7(-/-)$ and $10(+/+)$. Tet $1^{144-/-}$ mice showed reduced overall crouching time (effect of genotype: $F(1,15)=5.357, P=0.035$ ) (middle panel). Tet $7^{\text {1e4- / }}$ mice showed increased aggressive interactions in the first 15 minutes of the

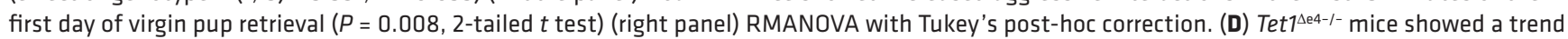
to reduced preference in training, short-term, and long-term memory in the object exploration (effect of genotype: $F[1,48]=3.877, P=0.055 ;$ effect of time: $\mathrm{F}[2,96]=0.035, P=0.053)$. Train, training phase; STM, short-term memory; LTM, long-term memory. $n=21(-/-)$ and $29(+/+)$. RMANOVA.

Tet $1^{1 e 4-1-}$ mice display impaired response to OXTR agonist stimulation but normal synaptic plasticity in the hippocampus. The reduced expression of Oxtr and the similar behavioral phenotype of Tet $1^{1 \text { set-I- }}$ to Oxtr ${ }^{\prime-}$ mice prompted us to examine the functional deficits specific to Oxtr. It has been shown that OXTR agonist (TGOT) increases the frequency and amplitude of spontaneous inhibitory postsynaptic currents (sIPSCs) onto hippocampal CA1 pyramidal neurons $(72,73)$. We speculated that if the reduced expression of Oxtr in Tet $1^{1 e 4-/-}$ neurons affected the function of OXTR, the response to oxytocin in pyramidal cells could be impaired. Thus, we recorded sIPSC from CA1 pyramidal cells at baseline, as well as in the presence of TGOT stimulation. The mean frequency at baseline for Tet $1^{\text {teq-/- }}(4.16 \pm 0.67 \mathrm{~Hz})$ is borderline lower than that of $\operatorname{Tet}^{+/+}(2.47 \pm 0.48 \mathrm{~Hz})$ (Figure 6A). The increase of sIPSC frequency in response to TGOT in Tet $1^{t e 4-/-}$ is similar to that of Tet $1^{+/+}$mice. The amplitude of sIPSC of Tet $1^{\text {teq-/- }}$ neurons $(26.56 \pm 6.78 \mathrm{pA})$ is comparable with that of $T e t 1^{+/+}$mice $(20.36 \pm 2.19 \mathrm{pA})$. However, the amplitude of sIPSC in response to TGOT in Tet $1^{-1-}$ neurons is significantly diminished compared with that of $\operatorname{Tet}^{+/+}$(Figure 6B). 
A previous study reports the impaired long-term synaptic plasticity in hippocampus of mutant mice lacking both DNA methyltransferases Dnmt1 and Dnmt3a in postnatal brain, suggesting that DNA methylation is essential for synaptic function (74). We then examined whether deficiency of Tet 1 may also affect synaptic function. The baseline synaptic transmission (Figure 6C) and the presynaptic function measured by paired pulse facilitation (PPF) and fiber volley are not affected (Figure 6, D and E). In contrast to the impaired longterm potentiation (LTP) in the hippocampus of Dnmt1 and Dnmt3a mutant mice, the synaptic plasticity as measured by hippocampal LTP in hippocampal CA synapses were not altered in Tet $1^{\text {tet-I- }}$ mice (Figure 6F).

\section{Discussion}

OXTR, the receptor for oxytocin, is a 9-amino acid neuropeptide produced in the brain and functions in both peripheral reproductive tissues and in the brain $(2,3)$. The molecular mechanism regulating the dynamic expression of $O X T R$ in response to different physiological stimuli has not been characterized. Our discovery of the complex transcriptional regulation and hypermethylation of Oxtr in $\mathrm{Tet} 1^{\mathrm{\Delta e} 4-/-}$ mice provide insight into transcriptional regulation of Oxtr and support that Oxtr is epigenetically regulated and that hypermethylation of $\mathrm{CpG}$ island correlates with the reduced expression of Oxtr isoform in vivo.

Previous in vitro studies have suggested that 2 regions may implicate DNA methylation in expression of Oxtr. First, the methylation of 2 CpG sites ( -932 and -733 ; the canonical TSS within the exon 1 is numbered as +1 ) in a region overlapping with the BS1 analyzed in this study has been shown to be negatively correlated with the expression of Oxtr in the mouse 4T1 cell line and brains $(10,11)$. Second, the methylation of CpG site in a 450 bp region (MT2) downstream of 5' canonical TSS that overlaps the homologous BS2 region is important for decreased expression of human OXTR in vitro (9). In brain regions analyzed in Tet $1^{\Delta e 4-/-}$ mice, we did not find a significant methylation difference for BS1, but we did discover a significant difference in the BS2 and BS3 regions. Consistent with these results, the full-length isoform Oxtr- $A$, for which the transcript begins in the BS1 region, was unaltered in Tet $1^{1 \mathrm{e} 4-/-}$ mice. Isoform $O x t r-B$, a short isoform initiated from an alternative downstream promoter in BS2-BS3 region appeared most affected by the hypermethylation.

The human -934 (The A in the ATG codon is denoted as +1 position) and adjacent CG sites, regions that have been studied in many epigenetic-associated studies in human psychiatric disorders, are homologous to the hypermethylated region in the BS2 in Tet $1^{\text {se4-/- }}$ mice based on the in silico analysis of genomic position. However, it is difficult to pinpoint the exact CG site in mice that is equivalent to the human $\mathrm{CpG}$ -934 CG site. The discovery of potentially novel mRNA isoforms in our study presents many questions about the transcriptional regulation of Oxtr. It remains to be determined whether each mRNA isoform has brain region- or cell type-specific expression or if each isoform is translated into a protein and how the methylation state of individual CG sites regulate isoform-specific Oxtr expression. Nevertheless, our finding raises interesting questions about whether each isoform may possess a specific function in the developing or adult brain.

Our Tet1-KO mouse with a deletion of exon 4 is potentially novel. While we recognize the reports of other 3 different lines of Tet 1 mutant mice (42-44), we believe that our line of Tet 1 mutant mouse still has significant value for future study of TET1 function in vivo because of possible alternative splicing of Tet 1 suggested by a recent report (45) and in silico data. The dysregulation of Npas 4 is consistent with the finding from the Tet1 $\Delta \mathrm{e} 5$ line of Tet1 mutant mice (37). The reduced expression of Oxtr in Tet $1^{\text {se4-/- }}$ mice is potentially novel and has not been addressed in other lines of Tet1 mutant mice. Our study suggests that early prenatal development is a critical period for TET1 function, as was also reported in a previous in vitro study (29) and a recent report indicating a lineage-specific role of TET1 in embryonic development (75). The Oxtr CpG island is hypermethylated in the E14.5 cerebrum, as well as mesodermal and endodermal tissues, but not in ESCs. The most plausible explanation would be that, without TET1, the Oxtr CpG island becomes hypermethylated during the onset of de novo methylation between E4.5-7.5 and the aberrant methylation is then propagated in every cell from that point. Rather than a role in active demethylation, the hypermethylation of Oxtr in Tet1 mutant mice supports a role for TET1 in preventing de novo methylation, as has previously been suggested $(29,76)$. In addition, the finding of intermediate hypermethylation of Oxtr in heterozygous Tet $1^{\text {te4+/- }}$ mice suggests that proper establishment of DNA methylation during development is sensitive to levels of TET1.

The role of oxytocin and OXTR in the neural circuitry involved in maternal care and social behavior has been well documented (77-81). In mice, both $\mathrm{Oxtr}^{-1}$ dams and $\mathrm{Oxtr}^{-1}$ virgin females take longer to retrieve pups and spend less time crouching over them in the pup retrieval test (68). In addition to aberrant maternal 

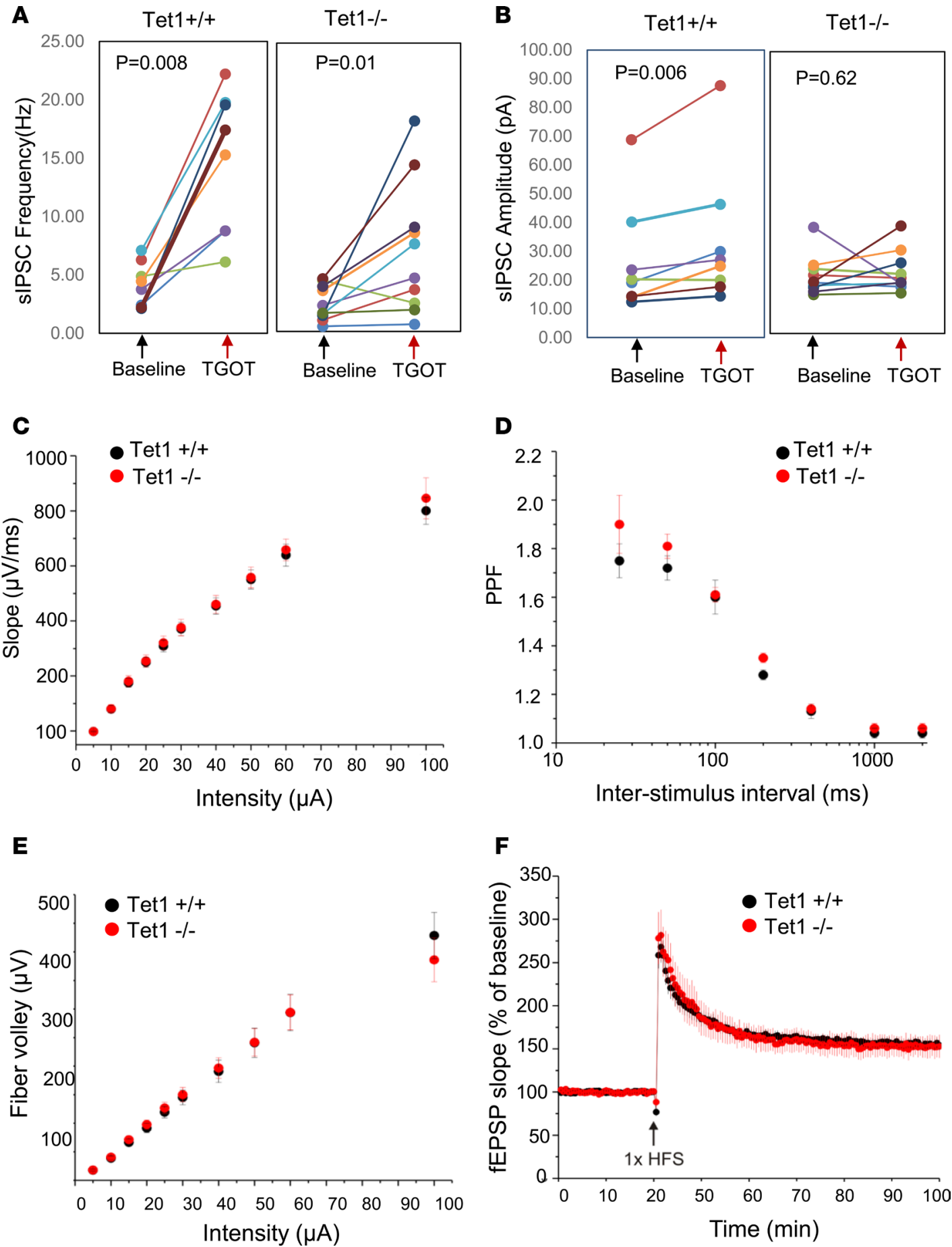

Figure 6. Tet1 ${ }^{\text {se4-/- }}$ mice display impaired response to OXTR agonist stimulation but normal synaptic plasticity in the hippocampus. (A) The frequency of

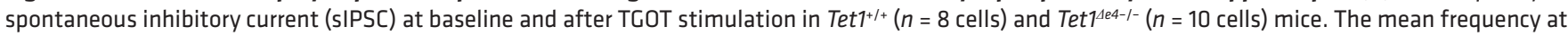

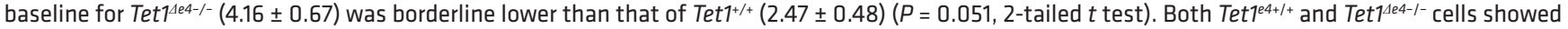
the significant increased frequency in response to TCOT stimulation (Wilcoxon signed ranks test; $P=0.008$ for $+/+$ and $P=0.01$ for $-/-$ ). (B) The amplitude of spontaneous inhibitory current (sIPSC) at baseline and after TCOT stimulation in Tet $7^{24+/+}\left(n=8\right.$ cells) and Tet $1^{1 e^{4-I-}}(n=10$ cells) mice. The amplitude

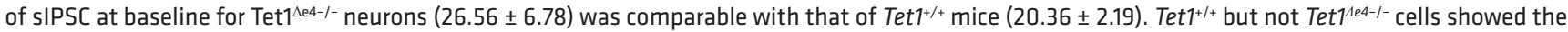
significant increased amplitude in response to TGOT stimulation (Wilcoxon signed ranks test; $P=0.006$ for $+/+$ and $P=0.65$ for $-/-$ ). (C) Baseline synaptic transmission not different in hippocampal CA1 of Tet1 1e4-I- mice. ( $n=8[-/-]$ slices from 5 mice; $n=11[+/+]$ slices from 6 mice of $6-8$ weeks old). (D) Paired pulse facilitation (PPF) not different in hippocampal CA1 of Tet ${ }^{1 \text { te4-I- }}$ mice indicating normal presynaptic function. ( $n=8$ [-/-] slices from 5 mice; $n=11$ $[+/+]$ slices from 6 mice of $6-8$ weeks old). (E) Fiber volley not different in hippocampal CA1 of Tet $1^{\text {te4-I- }}$ mice indicating normal presynaptic function ( $n=8$ [-/-] slices from 5 mice; $n=11[+/+]$ slices from 6 mice of $6-8$ weeks old). (F) LTP in CA1 of Tet $1^{\text {se- }-/-}$ was not different from Tet $1^{+/+}(+/+, 11$ slices from 6 mice; LTP, $156 \% \pm 6 \%$; -/-, 8 slice from 5 mice; LTP, 153\% $\pm 12 \%$; 2-tailed $t$ test). Arrow indicates the time of stimulation (HFS, $100 \mathrm{~Hz}, 1$ second). 
behavior, $\mathrm{Oxtr}^{-1-}$ males are more aggressive in the resident intruder test demonstrated by an increase in the number and duration of attacks (68). Our findings of increased threatening behaviors and decreased maternal care in $\mathrm{Tet}^{\mathrm{1 \textrm {e } 4 - /}}$ mice recapitulate the findings observed in $\mathrm{Oxtr}^{--}$mice and support the dysregulation of Oxtr in causing these behavioral deficits. The reduced response to oxytocin receptor agonist (TGOT) stimulation in TET1-deficient hippocampal neurons provides further support for impaired function of OXTR in the brain of Tet $1^{\text {se-1- }}$ mice. However, it may be noted that, in addition to downregulation of Oxtr, there are other dysregulated genes in our Tet $1^{\text {set/- }}$ mice that may confound the interpretation of behavioral phenotypes; therefore, further studies such as reexpression of OXTR in select brain regions of Tet $1^{\text {te4-1- }}$ mice may be warranted.

$O X T R$ has been the focus of extensive genetic and epigenetic studies for its role in psychiatric diseases or morbid behaviors in humans. There are more than 20 studies focusing on analysis of $5 \mathrm{mC}$ in the different CpG sites within the CpG island in humans (Supplemental Figure 10) (14, 17, 54-56, 82-92). The regions equivalent to the hypermethylated BS1 and BS2 in Tet1 mutant mice have been the focus in these studies. In most studies, only select $\mathrm{CpG}$ sites, ranging from 1-20, within the CpG island are analyzed $(55,93)$. Significant associations have been reported between OXTR DNA hypermethylation in these CpG sites and a wide spectrum of morbid behaviors and neuropsychiatric disease, such as ASD, schizophrenia, bipolar, OCD, and postpartum depression. Specifically, hypermethylation of a specific CpG site -934 of OXTR is reported in human postmortem autism brain tissues and is associated with schizophrenia, social response, and perception to anger and fear in human brains $(14,55,56,93)$. The hypermethylation is typically associated with the reduced expressions of OXTR in the majority of the studies, but the opposite directions are also observed in a few CG sites. Until now, any mechanism underlying the change of methylation of OXTR in these studies was entirely unknown. Our finding provides the first evidence to our knowledge of a potential mechanism for $O X T R$ hypermethylation and suggests that TET1 implicates the underlying mechanism of hypermethylation of human $O X T R$ found in neuropsychiatric diseases.

The overall transcriptional regulation and isoform structure between human and mice are predicted to be highly conserved $(5,6,67)$. The Oxtr transcript structure suggests a necessity of performing a reanalysis of results from existing epigenetic studies of OXTR in human, as the differences in methylation at unique $\mathrm{CpG}$ sites are likely to have different functional consequence on isoform-specific $O X T R$ expression. In addition, multiple human studies have found strong associations between genomic variants in $O X T R$ and ASD $(13,94-96)$. Our findings provide valuable information to reevaluate whether different OXTR isoforms are affected in these studies. Of particular interest, our finding that Oxtr is hypermethylated in tissues from all germ layers supports the use of peripheral blood in neurodevelopmental studies. Furthermore, our finding that TET1 has a dosage-sensitive effect on Oxtr methylation is particularly interesting, considering that heterozygous TET1 mutation has been reported in individuals with ASD (97). Altogether, our discovery of the involvement of TET1 in the epigenetic regulation of Oxtr and the complex transcriptional structure of Oxtr provide insights into understanding the role of OXTR in brain function and in neuropsychiatric disorders. The region-specific hypermethylation in the $\mathrm{CpG}$ island and isoform-specific reduction of Oxtr in Tet1 mutant mice will guide the experimental design for future OXTR epigenetic and genetic studies in human neuropsychiatric disorders.

\section{Methods}

Detailed experimental procedures can be found in Supplemental Methods.

Generation of Tet $1^{1 e 4-1-}$ mice. For gene targeting and Southern analysis, we created a floxed construct of Tet 1 exon 4 using the recombineering method described by Liu and colleagues (98). The 129SvEv BAC clone (bMQ-258L12) covering the Tet1 gene was first identified in silico using the Ensembl mouse genome browser (www.ensembl.org) and obtained from Geneservice (www.geneseservice.uk.com) (99). A 13.7-kb genomic fragment containing exon 4 of the Tet1 gene was retrieved into a plasmid from the BAC clone. A neomycin (neo) selectable marker flanked by flippase recognition target (FRT) sites was inserted into the plasmid. Two loxP sites were inserted into the plasmid flanking exon 4 . The $5^{\prime}$ loxP was introduced at the genomic site of $62,304,376 \mathrm{bp}$, and the $3^{\prime}$ loxP/neo cassette at 62,300,243 bp (www.genome.ucsc.edu; mm9). The finalized plasmid was linearized with NotI and electroporated into R1 129/Sv mouse ESCs at the Duke Neurotransgenic Laboratory. Neo-resistant colonies were picked after 7-8 days of selection, and correctly recombinant clones were identified by Southern blot analysis using both a $5^{\prime}$ and $3^{\prime}$ probe. Tet $1^{e 4+/ f}$ ESCs were injected into blastocysts to produce the chimeric male mice. These were bred with CMV-Cre females (The Jackson Laboratory, stock no. 006054) to obtain germline transmission of the Tet1-deleted allele. Tet $1^{\text {te4+/- }}$ mice were 
backcrossed to C57BL/6J mice (The Jackson Laboratory, stock no. 000664) for more than 6 generations (N6) before molecular experiments and behavioral analysis. We segregated out a naturally occurring mutation in the Disc1 gene in the 129R1 mouse strain from which ESCs were derived during the backcrossing. Multiple cohorts of Tet $1^{\text {se4-/- }}$ mice and WT control $\left(\right.$ Tet $\left.1^{+/+}\right)$littermates were obtained from heterozygous breeding. All mice used for molecular experiments were between 5-10 weeks of age and were between 2-6 months for behavioral tests. Mice were sex-matched for all experiments.

Bisulfite sequencing. DNA (2 $\mu \mathrm{g})$ from each sample was bisulfite-converted using the EpiTect bisulfite kit (Qiagen) according to the manufacturer's protocol. Bisulfite-converted DNA was amplified in 40 cycles using EpiMark Hot Start Taq DNA polymerase (New England Biolabs). Specific primers and annealing temperatures are listed in Supplemental Methods. PCR fragments were recovered by gel excision and cloned into the pGEM-T easy vector (Promega). Typically, 16 clones from each PCR product were sequenced with M13R primers at Beckman Coulter Genomics or EtonBio. A minimum of 10-12 clones with high-quality sequencing was obtained for each sample. Only the sequence products with bisulfite $\mathrm{C}-\mathrm{T}$ conversion rate $>99 \%$ were included for the data analysis, and we were able to achieve this conversion rate in most of experiments performed. Sequencing reads were analyzed using the online platform BISMA using default analysis parameters: Lower threshold conversion rate 99\%, lower threshold sequence identity $90 \%$, upper threshold of $\mathrm{N}$-sites at cytosine position $20 \%$, upper threshold gaps allowed $20 \%$ (100).

Field potential recording. Six- to 8 week-old mice were used for the LTP experiment. The hippocampus was cut in transverse sections at $400 \mu \mathrm{m}$ in the slicing solution containing $75 \mathrm{mM}$ sucrose, $87 \mathrm{mM} \mathrm{NaCl}$, $2.5 \mathrm{mM} \mathrm{KCl}, 1.25 \mathrm{mM} \mathrm{NaH}_{2} \mathrm{PO}_{4}, 26 \mathrm{mM} \mathrm{NaHCO}_{3}, 10 \mathrm{mM}$ glucose, $7 \mathrm{mM} \mathrm{MgCl}$, and $0.5 \mathrm{mM} \mathrm{CaCl}_{2}$. Slices were recovered at least 2 hours at $30^{\circ} \mathrm{C}$ in ACSF containing $124 \mathrm{mM} \mathrm{NaCl}, 3 \mathrm{mM} \mathrm{KCl}, 1.25 \mathrm{mM}$ $\mathrm{NaH}_{2} \mathrm{PO}_{4}, 26 \mathrm{mM} \mathrm{NaHCO}_{3}, 10 \mathrm{mM}_{\text {glucose }} 1 \mathrm{mM} \mathrm{MgCl}_{2}$, and $2 \mathrm{mM} \mathrm{CaCl}_{2}$. In the submersion recording chamber $\left(30^{\circ} \mathrm{C}\right)$, a glass recording electrode (1-3 M $)$ filled with artificial cerebrospinal fluid (ACSF) was placed in CA1 radiatum. At baseline, stimulations (200 $\mu$ sec, DS301 or Isoflex) were applied to the Schaffer collaterals every 30 seconds with a concentric bipolar tungsten electrode. The input-output relationship was obtained from increasing stimulation intensities (intervals of 5, 10, 15, 20, 25, 30, 40, 50, 60, $100 \mu \mathrm{A}$ ). Paired-pulse ratios were obtained from the field excitatory postsynaptic potential (fEPSP) slope of the second to the first stimulus at given interstimulus intervals $(25-2,000 \mathrm{~ms})$. For LTP, the stimulus strength was adjusted to evoke a fEPSP at half of the maximal response. After recording a stable baseline for 20 minutes (less than $5 \%$ drift), high-frequency stimulation $(100 \mathrm{~Hz}, 1$ second) was applied. For the next 60 minutes, activity to a single pulse was recorded. The slope at 55-60 minutes was compared with the preconditioning baseline response (last 5 minutes of baseline). Values are expressed as means \pm SEM.

Whole cell recording. Hippocampi from mice ages 2-3 months were cut in transverse section at $300 \mu \mathrm{m}$ in a slicing solution containing $75 \mathrm{mM}$ sucrose, $87 \mathrm{mM} \mathrm{NaCl}, 2.5 \mathrm{mM} \mathrm{KCl}, 1.25 \mathrm{mM} \mathrm{NaH}_{2} \mathrm{PO}_{4}, 26 \mathrm{mM}$ $\mathrm{NaHCO}_{3}, 10 \mathrm{mM}$ glucose, $7 \mathrm{mM} \mathrm{MgCl}_{2}$, and $0.5 \mathrm{mM} \mathrm{CaCl}_{2}$. Slices were incubated for 30 minutes at $34^{\circ} \mathrm{C}$ in ACSF containing $124 \mathrm{mM} \mathrm{NaCl}, 3 \mathrm{mM} \mathrm{KCl}, 1.25 \mathrm{mM} \mathrm{NaH}_{2} \mathrm{PO}_{4}, 26 \mathrm{mM} \mathrm{NaHCO}_{3}, 10 \mathrm{mM}$ glucose, 1 $\mathrm{mM} \mathrm{MgCl}_{2}$, and $2 \mathrm{mM} \mathrm{CaCl}_{2}$. After 1 hour at room temperature, a slice was transferred to the submersion recording chamber $\left(30^{\circ} \mathrm{C}\right)$.

Individual CA1 pyramidal cells were visualized with an upright microscope (Zeiss, Examiner.D1). The patch pipette (4-6 M $\Omega$ ) was filled with an internal solution containing $40 \mathrm{mM} \mathrm{CsCl,} 90 \mathrm{mM}$ K-gluconate, $1.8 \mathrm{mM} \mathrm{NaCl}, 1.7 \mathrm{mM} \mathrm{MgCl}_{2}, 3.5 \mathrm{mM} \mathrm{KCl}, 0.05 \mathrm{mM}$ EGTA, $10 \mathrm{mM}$ HEPES, $2 \mathrm{mM} \mathrm{MgATP}, 0.4$ $\mathrm{Na}_{2}$ GTP, $10 \mathrm{mM}$ phosphocreatine ( $\mathrm{pH} 7.3$ ), and $290 \mathrm{mOsm}$. Signals were amplified and filtered at $3 \mathrm{k} \mathrm{Hz}$ with Bessel filter (MultiClamp 700B, Molecular Devices) and were then digitized at $10 \mathrm{kHz}$ with a Digidata 1440A (Molecular Devices).

Spontaneous inhibitory currents (sIPSC) was recorded at $-70 \mathrm{mV}$ in the presence of NBQX $(10 \mu \mathrm{M})$ and D-AP5 (50 $\mu \mathrm{M})$. TGOT (1 $\mu \mathrm{M}, 2$ minutes; Bachem) was bath applied. sIPSC amplitude and frequency were detected using Minianalysis (Synaptosoft) for 2 minutes each before TGOT and during peak sIPSC activity after TGOT.

Accession numbers. Oxtr mRNA isoform sequencing data has been deposited in GenBank under accession numbers KU686795-KU686801. RNA-seq and DNA methylome data have been deposited in GEO under accession number GSE99674.

Statistics. The data were analyzed with SPSS 21 (SPSS Inc.) or GraphPad Prism and expressed as mean \pm SEM. Simple comparisons between Tet $1^{1 e 4-/-}$ and $T e t 1^{+/+}$mice without regard to sex were conducted with independent $t$ tests (Student's $t$ test, 2-tailed). For behavioral data, when comparisons between genotypes 
were made for within-subject measurements across different phases of the same test (e.g., test days, locations within a test arena, or different intensities of stimuli), the data were analyzed with repeated measures 2-way ANOVA (RMANOVA). For 2-way RMANOVAs, a Tukey's correction for multiple comparisons was applied for post-hoc analysis. Statistical significance was defined as $P<0.05$. Sample sizes were based on previous experience with similarly designed experiments.

Study approval. All experiments were conducted with protocols approved by the IACUC at Duke University. The animal studies presented in this paper have been approved by Duke Animal Care Committee with protocol number of A137-17-06.

\section{Author contributions}

AJT, XL, XK, and YJ conceived the experiments. AJT, XL, and XW created the Tet1 mice and ESCs. AJT, MWT, XC, XW, SX, and YK performed the molecular experiments. PW analyzed the RNA-seq data. WZ and WX performed the methylome experiment. AJT, ALB, LJD, and SKS performed behavioral experiments and ALB performed the statistical analyses. LC performed the slice physiology. AJT, MWT, SG, and YJ wrote the manuscript.

\section{Acknowledgments}

We would like to thank Ute Hochgeschwender for assistance with mouse production, William Wetsel and Ramona Rodriguiz for help with behavioral tests, Anne West for Bdnf and Homerla primers, and Angel Peterchev for assistance with the ECS experiment. YHJ was supported by NIH grants MH098114, MH104316, HD077197, HD088626, and HD088007, as well as awards from Autism Speaks, and the Ruth K. Broad Foundation. XW was supported by a fellowship from the PMS Foundation. ALB was supported by the Ruth K. Broad and Autism Science Foundations and Duke MSTP T32 GM007171. MWT was supported by Duke Genomics and Genetics training grant T32 GM007754 and Marcy Speer Memorial Fellowship.

Address correspondence to: Yong-hui Jiang, Departments of Pediatrics and Neurobiology, Duke University School of Medicine, GSRB1 4004, 905 S. LaSalle Street, Durham, North Carolina 27710, USA. Phone: 919.681.2789; Email:yong-hui.jiang@duke.edu.

1. Grinevich V, Knobloch-Bollmann HS, Eliava M, Busnelli M, Chini B. Assembling the Puzzle: Pathways of Oxytocin Signaling in the Brain. Biol Psychiatry. 2016;79(3):155-164.

2. Insel TR. The challenge of translation in social neuroscience: a review of oxytocin, vasopressin, and affiliative behavior. Neuron. 2010;65(6):768-779.

3. Kumsta R, Heinrichs M. Oxytocin, stress and social behavior: neurogenetics of the human oxytocin system. Curr Opin Neurobiol. 2013;23(1):11-16.

4. Gimpl G, Fahrenholz F. The oxytocin receptor system: structure, function, and regulation. Physiol Rev. 2001;81(2):629-683.

5. Inoue T, et al. Structural organization of the human oxytocin receptor gene. J Biol Chem. 1994;269(51):32451-32456.

6. Kubota Y, et al. Structure and expression of the mouse oxytocin receptor gene. Mol Cell Endocrinol. 1996;124(1-2):25-32.

7. Mitre M, et al. A Distributed Network for Social Cognition Enriched for Oxytocin Receptors. J Neurosci. 2016;36(8):2517-2535.

8. Vaidyanathan R, Hammock EA. Oxytocin receptor dynamics in the brain across development and species. Dev Neurobiol. 2017;77(2):143-157.

9. Kusui C, et al. DNA methylation of the human oxytocin receptor gene promoter regulates tissue-specific gene suppression. Biochem Biophys Res Commun. 2001;289(3):681-686.

10. Mamrut S, et al. DNA methylation of specific $\mathrm{CpG}$ sites in the promoter region regulates the transcription of the mouse oxytocin receptor. PLOS ONE. 2013;8(2):e56869.

11. Harony-Nicolas H, Mamrut S, Brodsky L, Shahar-Gold H, Barki-Harrington L, Wagner S. Brain region-specific methylation in the promoter of the murine oxytocin receptor gene is involved in its expression regulation. Psychoneuroendocrinology. 2014;39:121-131.

12. Heinrichs M, Baumgartner T, Kirschbaum C, Ehlert U. Social support and oxytocin interact to suppress cortisol and subjective responses to psychosocial stress. Biol Psychiatry. 2003;54(12):1389-1398.

13. LoParo D, Waldman ID. The oxytocin receptor gene (OXTR) is associated with autism spectrum disorder: a meta-analysis. $M o l$ Psychiatry. 2015;20(5):640-646.

14. Gregory SG, et al. Genomic and epigenetic evidence for oxytocin receptor deficiency in autism. BMC Med. $2009 ; 7: 62$.

15. Elagoz Yuksel M, Yuceturk B, Karatas OF, Ozen M, Dogangun B. The altered promoter methylation of oxytocin receptor gene in autism. J Neurogenet. 2016;30(3-4):280-284.

16. Francis SM, Kim SJ, Kistner-Griffin E, Guter S, Cook EH, Jacob S. ASD and Genetic Associations with Receptors for Oxytocin and Vasopressin-AVPR1A, AVPR1B, and OXTR. Front Neurosci. 2016;10:516.

17. Watanabe T, et al. Oxytocin receptor gene variations predict neural and behavioral response to oxytocin in autism. Soc Cogn Affect Neurosci. 2017;12(3):496-506. 
18. Maud C, Ryan J, McIntosh JE, Olsson CA. The role of oxytocin receptor gene (OXTR) DNA methylation (DNAm) in human social and emotional functioning: a systematic narrative review. BMC Psychiatry. 2018;18(1):154.

19. Cimarelli G, Virányi Z, Turcsán B, Rónai Z, Sasvári-Székely M, Bánlaki Z. Social Behavior of Pet Dogs Is Associated with Peripheral OXTR Methylation. Front Psychol. 2017;8:549.

20. Baker M, et al. Early rearing history influences oxytocin receptor epigenetic regulation in rhesus macaques. Proc Natl Acad Sci USA. 2017;114(44):11769-11774.

21. Kriaucionis S, Heintz N. The nuclear DNA base 5-hydroxymethylcytosine is present in Purkinje neurons and the brain. Science. 2009;324(5929):929-930.

22. Tahiliani M, et al. Conversion of 5-methylcytosine to 5-hydroxymethylcytosine in mammalian DNA by MLL partner TET1. Science. 2009;324(5929):930-935.

23. Ito S, et al. Tet proteins can convert 5-methylcytosine to 5-formylcytosine and 5-carboxylcytosine. Science. 2011;333(6047):1300-1303.

24. He YF, et al. Tet-mediated formation of 5-carboxylcytosine and its excision by TDG in mammalian DNA. Science. 2011;333(6047):1303-1307.

25. Szwagierczak A, Bultmann S, Schmidt CS, Spada F, Leonhardt H. Sensitive enzymatic quantification of 5-hydroxymethylcytosine in genomic DNA. Nucleic Acids Res. 2010;38(19):e181.

26. Wossidlo M, et al. 5-Hydroxymethylcytosine in the mammalian zygote is linked with epigenetic reprogramming. Nat Commun $2011 ; 2: 241$.

27. Ito S, D'Alessio AC, Taranova OV, Hong K, Sowers LC, Zhang Y. Role of Tet proteins in $5 \mathrm{mC}$ to 5 hmC conversion, ES-cell self-renewal and inner cell mass specification. Nature. 2010;466(7310):1129-1133.

28. Koh KP, et al. Tet1 and Tet2 regulate 5-hydroxymethylcytosine production and cell lineage specification in mouse embryonic stem cells. Cell Stem Cell. 2011;8(2):200-213.

29. Xu Y, et al. Genome-wide regulation of $5 \mathrm{hmC}, 5 \mathrm{mC}$, and gene expression by Tet 1 hydroxylase in mouse embryonic stem cells Mol Cell. 2011;42(4):451-464.

30. Globisch D, et al. Tissue distribution of 5-hydroxymethylcytosine and search for active demethylation intermediates. PLoS ONE 2010;5(12):e15367.

31. Münzel M, et al. Quantification of the sixth DNA base hydroxymethylcytosine in the brain. Angew Chem Int Ed Engl. 2010;49(31):5375-5377.

32. Bachman M, Uribe-Lewis S, Yang X, Williams M, Murrell A, Balasubramanian S. 5-Hydroxymethylcytosine is a predominantly stable DNA modification. Nat Chem. 2014;6(12):1049-1055.

33. Song CX, et al. Selective chemical labeling reveals the genome-wide distribution of 5-hydroxymethylcytosine. Nat Biotechnol. 2011;29(1):68-72.

34. . Nature Reviews Genetics. From the editors. Nat Rev Genet. 2011;12(8):517.

35. Bochtler M, Kolano A, Xu GL. DNA demethylation pathways: Additional players and regulators. Bioessays. 2017;39(1):1-13.

36. Meadows JP, et al. DNA methylation regulates neuronal glutamatergic synaptic scaling. Sci Signal. 2015;8(382):ra61.

37. Rudenko A, et al. Tet1 is critical for neuronal activity-regulated gene expression and memory extinction. Neuron. 2013;79(6):1109-1122.

38. Yao B, Christian KM, He C, Jin P, Ming GL, Song H. Epigenetic mechanisms in neurogenesis. Nat Rev Neurosci. 2016;17(9):537-549.

39. Yao B, Jin P. Unlocking epigenetic codes in neurogenesis. Genes Dev. 2014;28(12):1253-1271.

40. Kaas GA, et al. TET1 controls CNS 5-methylcytosine hydroxylation, active DNA demethylation, gene transcription, and memory formation. Neuron. 2013;79(6):1086-1093.

41. Kumar D, et al. Tet1 Oxidase Regulates Neuronal Gene Transcription, Active DNA Hydroxy-methylation, Object Location Memory, and Threat Recognition Memory. Neuroepigenetics. 2015;4:12-27.

42. Zhang RR, et al. Tet1 regulates adult hippocampal neurogenesis and cognition. Cell Stem Cell. 2013;13(2):237-245

43. Dawlaty MM, et al. Tet1 is dispensable for maintaining pluripotency and its loss is compatible with embryonic and postnatal development. Cell Stem Cell. 2011;9(2):166-175

44. Yamaguchi S, et al. Tet1 controls meiosis by regulating meiotic gene expression. Nature. 2012;492(7429):443-447.

45. Zhang W, et al. Isoform Switch of TET1 Regulates DNA Demethylation and Mouse Development. Mol Cell. 2016;64(6):1062-1073.

46. Heinz S, et al. Simple combinations of lineage-determining transcription factors prime cis-regulatory elements required for macrophage and B cell identities. Mol Cell. 2010;38(4):576-589.

47. Wu H, et al. Dual functions of Tet1 in transcriptional regulation in mouse embryonic stem cells. Nature. 2011;473(7347):389-393

48. Tan L, Shi YG. Tet family proteins and 5-hydroxymethylcytosine in development and disease. Development. 2012;139(11):1895-1902.

49. Zhu X, et al. Role of Tet1/3 Genes and Chromatin Remodeling Genes in Cerebellar Circuit Formation. Neuron. 2016;89(1):100-112.

50. West AE, Greenberg ME. Neuronal activity-regulated gene transcription in synapse development and cognitive function. Cold Spring Harb Perspect Biol. 2011;3(6):a005744.

51. Nibuya M, Morinobu S, Duman RS. Regulation of BDNF and trkB mRNA in rat brain by chronic electroconvulsive seizure and antidepressant drug treatments. J Neurosci. 1995;15(11):7539-7547.

52. Brakeman PR, et al. Homer: a protein that selectively binds metabotropic glutamate receptors. Nature. 1997;386(6622):284-288.

53. Ramamoorthi K, et al. Npas4 regulates a transcriptional program in CA3 required for contextual memory formation. Science. 2011;334(6063):1669-1675.

54. Bell AF, et al. Interaction between oxytocin receptor DNA methylation and genotype is associated with risk of postpartum depression in women without depression in pregnancy. Front Genet. 2015;6:243.

55. Jack A, Connelly JJ, Morris JP. DNA methylation of the oxytocin receptor gene predicts neural response to ambiguous social stimuli. Front Hum Neurosci. 2012;6:280.

56. Puglia MH, Lillard TS, Morris JP, Connelly JJ. Epigenetic modification of the oxytocin receptor gene influences the perception of anger and fear in the human brain. Proc Natl Acad Sci USA. 2015;112(11):3308-3313. 
57. ENCODE Project Consortium. An integrated encyclopedia of DNA elements in the human genome. Nature. 2012;489(7414):57-74.

58. Zhang H, Zhang X, Clark E, Mulcahey M, Huang S, Shi YG. TET1 is a DNA-binding protein that modulates DNA methylation and gene transcription via hydroxylation of 5-methylcytosine. Cell Res. 2010;20(12):1390-1393.

59. Hon GC, et al. Epigenetic memory at embryonic enhancers identified in DNA methylation maps from adult mouse tissues. Nat Genet. 2013;45(10):1198-1206.

60. Xi Y, Li W. BSMAP: whole genome bisulfite sequence MAPping program. BMC Bioinformatics. 2009;10:232.

61. Kimura T, Tanizawa O, Mori K, Brownstein MJ, Okayama H. Structure and expression of a human oxytocin receptor. Nature. 1992;356(6369):526-529.

62. Cedar H, Bergman Y. Linking DNA methylation and histone modification: patterns and paradigms. Nat Rev Genet. 2009;10(5):295-304

63. Yue F, et al. A comparative encyclopedia of DNA elements in the mouse genome. Nature. 2014;515(7527):355-364

64. Bernstein BE, et al. A bivalent chromatin structure marks key developmental genes in embryonic stem cells. Cell. 2006;125(2):315-326.

65. Lin Y, et al. Activity-dependent regulation of inhibitory synapse development by Npas4. Nature. 2008;455(7217):1198-1204

66. Caldwell HK, Aulino EA, Freeman AR, Miller TV, Witchey SK. Oxytocin and behavior: Lessons from knockout mice. Dev Neurobiol. 2017;77(2):190-201.

67. Feldman R, Monakhov M, Pratt M, Ebstein RP. Oxytocin Pathway Genes: Evolutionary Ancient System Impacting on Human Affiliation, Sociality, and Psychopathology. Biol Psychiatry. 2016;79(3):174-184.

68. Takayanagi Y, et al. Pervasive social deficits, but normal parturition, in oxytocin receptor-deficient mice. Proc Natl Acad Sci USA. 2005;102(44):16096-16101.

69. Noirot E. Changes in responsiveness to young in the adult mouse. V. Priming. Anim Behav. 1969;17(3):542-546.

70. Stolzenberg DS, Stevens JS, Rissman EF. Experience-facilitated improvements in pup retrieval; evidence for an epigenetic effect. Horm Behav. 2012;62(2):128-135.

71. Lonstein JS, De Vries GJ. Sex differences in the parental behavior of rodents. Neurosci Biobehav Rev. 2000;24(6):669-686.

72. Zaninetti M, Raggenbass M. Oxytocin receptor agonists enhance inhibitory synaptic transmission in the rat hippocampus by activating interneurons in stratum pyramidale. Eur J Neurosci. 2000;12(11):3975-3984.

73. Owen SF, Tuncdemir SN, Bader PL, Tirko NN, Fishell G, Tsien RW. Oxytocin enhances hippocampal spike transmission by modulating fast-spiking interneurons. Nature. 2013;500(7463):458-462.

74. Feng J, et al. Dnmt1 and Dnmt3a maintain DNA methylation and regulate synaptic function in adult forebrain neurons. Nat Neurosci. 2010;13(4):423-430.

75. Khoueiry R, et al. Lineage-specific functions of TET1 in the postimplantation mouse embryo. Nat Genet. 2017;49(7):1061-1072

76. Jin C, et al. TET1 is a maintenance DNA demethylase that prevents methylation spreading in differentiated cells. Nucleic Acids Res. 2014;42(11):6956-6971.

77. Pedersen CA, Prange AJ. Induction of maternal behavior in virgin rats after intracerebroventricular administration of oxytocin. Proc Natl Acad Sci USA. 1979;76(12):6661-6665.

78. Pedersen CA, Vadlamudi SV, Boccia ML, Amico JA. Maternal behavior deficits in nulliparous oxytocin knockout mice. Genes Brain Behav. 2006;5(3):274-281.

79. Olazábal DE, Young LJ. Oxytocin receptors in the nucleus accumbens facilitate "spontaneous" maternal behavior in adult female prairie voles. Neuroscience. 2006;141(2):559-568.

80. Shahrokh DK, Zhang TY, Diorio J, Gratton A, Meaney MJ. Oxytocin-dopamine interactions mediate variations in maternal behavior in the rat. Endocrinology. 2010;151(5):2276-2286.

81. D’Cunha TM, King SJ, Fleming AS, Lévy F. Oxytocin receptors in the nucleus accumbens shell are involved in the consolidation of maternal memory in postpartum rats. Horm Behav. 2011;59(1):14-21.

82. Dadds MR, et al. Methylation of the oxytocin receptor gene and oxytocin blood levels in the development of psychopathy. Dev Psychopathol. 2014;26(1):33-40.

83. Cecil CA, et al. Environmental risk, Oxytocin Receptor Gene (OXTR) methylation and youth callous-unemotional traits: a 13-year longitudinal study. Mol Psychiatry. 2014;19(10):1071-1077.

84. Reiner I, Van IJzendoorn MH, Bakermans-Kranenburg MJ, Bleich S, Beutel M, Frieling H. Methylation of the oxytocin receptor gene in clinically depressed patients compared to controls: The role of OXTR rs53576 genotype. J Psychiatr Res. 2015;65:9-15.

85. Ziegler C, et al. Oxytocin receptor gene methylation: converging multilevel evidence for a role in social anxiety. Neuropsychopharmacology. 2015;40(6):1528-1538.

86. Unternaehrer E, et al. Dynamic changes in DNA methylation of stress-associated genes (OXTR, BDNF) after acute psychosocial stress. Transl Psychiatry. 2012;2:e150.

87. Grove TB, Burghardt KJ, Kraal AZ, Dougherty RJ, Taylor SF, Ellingrod VL. Oxytocin Receptor (OXTR) Methylation and Cog nition in Psychotic Disorders. Mol Neuropsychiatry. 2016;2(3):151-160.

88. Cappi C, et al. Epigenetic evidence for involvement of the oxytocin receptor gene in obsessive-compulsive disorder. BMC Neurosci. 2016;17(1):79.

89. Smearman EL, et al. Oxytocin Receptor Genetic and Epigenetic Variations: Association With Child Abuse and Adult Psychiatric Symptoms. Child Dev. 2016;87(1):122-134.

90. Chagnon YC, Potvin O, Hudon C, Préville M. DNA methylation and single nucleotide variants in the brain-derived neurotrophic factor (BDNF) and oxytocin receptor (OXTR) genes are associated with anxiety/depression in older women. Front Genet. 2015;6:230

91. Ebner NC, et al. Associations between oxytocin receptor gene (OXTR) methylation, plasma oxytocin, attachment across adulthood [published online ahead of print February 2, 2018]. Int J Psychophysiol. https://doi.org/10.1016/j.ijpsycho.2018.01.008.

92. Puglia MH, Connelly JJ, Morris JP. Epigenetic regulation of the oxytocin receptor is associated with neural response during selective social attention. Transl Psychiatry. 2018;8(1):116.

93. Rubin LH, et al. Sex and diagnosis specific associations between DNA methylation of the oxytocin receptor gene with emotion 
processing and temporal-limbic and prefrontal brain volumes in psychotic disorders. Biol Psychiatry Cogn Neurosci Neuroimaging. 2016;1(2):141-151.

94. Wu S, et al. Positive association of the oxytocin receptor gene (OXTR) with autism in the Chinese Han population. Biol Psychiatry. 2005;58(1):74-77.

95. Jacob S, Brune CW, Carter CS, Leventhal BL, Lord C, Cook EH. Association of the oxytocin receptor gene (OXTR) in Caucasian children and adolescents with autism. Neurosci Lett. 2007;417(1):6-9.

96. Lerer E, Levi S, Salomon S, Darvasi A, Yirmiya N, Ebstein RP. Association between the oxytocin receptor (OXTR) gene and autism: relationship to Vineland Adaptive Behavior Scales and cognition. Mol Psychiatry. 2008;13(10):980-988.

97. Parikshak NN, et al. Integrative functional genomic analyses implicate specific molecular pathways and circuits in autism. Cell. 2013;155(5):1008-1021.

98. Liu P, Jenkins NA, Copeland NG. A highly efficient recombineering-based method for generating conditional knockout mutations. Genome Res. 2003;13(3):476-484.

99. Adams DJ, et al. A genome-wide, end-sequenced 129Sv BAC library resource for targeting vector construction. Genomics. $2005 ; 86(6): 753-758$

100. Rohde C, Zhang Y, Reinhardt R, Jeltsch A. BISMA--fast and accurate bisulfite sequencing data analysis of individual clones from unique and repetitive sequences. BMC Bioinformatics. 2010;11:230. 